\title{
ANALOGY BETWEEN THE MODELLING OF PULLOUT IN SOLUTION SPINNING AND THE PREDICTION OF THE VORTEX SIZE IN CONTRACTION FLOWS
}

\author{
M.J.H. BULTERS \\ DSM Research Department of Material Development, P.O. Box 18, 6160 MD Geleen \\ (The Netherlands) \\ H.E.H. MEIJER \\ Eindhoven University of Technology, Faculty of Mechanical Engineering, \\ Polymer Technology. P.O. Box 513, 5600 MB Eindhoven (The Netherlands) \\ (Received March 26, 1990; in revised form July 10, 1990)
}

\begin{abstract}
Solutions of high molecular weight polymers in suitable solvents show a pronounced shear thinning behaviour combined with strong elastic effects and wall slippage. During spinning, these solutions are pulled out of the spinneret at a certain draw ratio. This process is studied. After surveying the equations governing the spinning process, attention is focussed on the position of the detachment point and its dependence on the solution properties and the processing conditions. A simple force balance at this point equilibrates the spinning tension (dominated by the elongational viscosity, the draw ratio and the strain rate) and the first normal stress difference (which depends on shear rate and die geometry). This analysis yields the upstream position of the detachment point. Intriguingly, the same analysis applies to the almost classical rheological problem of the entrance flow in a contraction. Following the same procedure, the size of the vortices can be predicted and their dependence on the Deborah number, provided that both the transient elongational viscosity and the first normal stress difference of the solution are known. In fact, the latter problem might be less complicated because the flow occurs under isothermal conditions. However, one has to neglect the two-dimensional character of the flow in order to reach analytical results.
\end{abstract}

Keywords: first normal stress difference; pullout; solution spinning; viscoelasticity; vortex size 


\section{Introduction}

The anisotropic nature of long polymer chains in melts or solutions gives rise to pronounced deviations from the Newtonian fluid behaviour. The rheology of these systems strongly depends on the high end tail of the molecular weight distribution [1], which hampers, amongst other things, the molecular interpretation of flow behaviour. Shear and uniaxial extensional flows can be modelled using factorable constitutive equations [2,3]. In the case of biaxial extensional flows, experimental data are rather scarce, see for example Ref. 4. The modelling of extensional flows is possible because of the simple geometry involved. An example is the fibre spinning process, which is now fairly well understood [5-8]. Also, film blowing $[9,10]$ and parison extrusion [11] have been modelled, with the necessary incorporation of the flow inside the die [10-14]. However, combined shear and extensional flows, e.g. contraction flows, are more complicated: numerical problems have to be solved and the quantitative agreement with experiments is not yet satisfactory [15], partly owing to the simplified constitutive equations used. Contraction flows not only serve as a measurement technique for an averaged elongational viscosity $[16,17]$ but have been made a pilot problem in the testing of the numerical analyses as condensed in different computer codes $[15,18,19]$. This choice is mainly based on the clarifying experiments of Boger and coworkers with model liquids in the $4: 1$ contraction [20-25].

In the search for the ultimate mechanical properties of polymers nowadays, solutions of ultrahigh molecular weight materials are spun, using conventional spinning techniques. A striking example is the so called 'gelspinning' process, named after the intermediate spun yarn which forms a gel during the crystallization of the polymer in the presence of a solvent [26,27]. Owing to the combination of ultrahigh molecular weight polymers and relatively high concentrations, viscoelastic effects are pronounced. This is illustrated by the need for extruders in the continuous preparation of homogeneous solutions [28]. In our research, the problem of spinning highly concentrated solutions of ultrahigh molecular weight polyethylene in decalin had to be solved in order to reach a stable process even at high throughputs. Because of the extreme character of the fluid, a careful design of the spinnerets proved to be important during the debottlenecking procedure. In the gelspinning process the solutions are pulled out of the capillary, at usual values of the draw ratio (see Fig. 6). More upstream, in the inflow of the capillary, vortices occur which at higher throughputs tend to become unstable, as reflected in a poor extrudate quality, and hence impose a limitation on the process.

In our analysis, pullout and the position of the detachment point are modelled and, as an extension thereof, the vortex size in the upstream 
contraction is predicted. The aim is a model which is as simple as possible, while still accounting for the dominant viscoelastic effects. Because of the problems with the rheological characterization of the solutions used in the gelspinning process, the model developed will be evaluated using experimental data on model liquids from the literature, notably the results of Sridhar and Gupta [29] on pullout, and of Binding and Walters [30] on the vortex size in contraction flows.

In Section 2 the classical viscous and viscoelastic modelling of spinning will be discussed briefly, in order to allow for a major simplification of the analysis. This result will be incorporated into the existing models of solution spinning with evaporating solvents, yielding expressions for the spinning force versus draw ratio, as a function of throughput and thermal boundary conditions.

In Section 3 the pullout phenomenon, which occurs in the gelspinning process as well as in the spinning of model liquids [29], will be presented. In the first case a combined isothermal and non-isothermal spinning process is found, dominated by the evaporation of solvent. Using the expressions from Section 2, attention is paid to the prediction of the position of the detachment point as a function of fluid properties (molecular weight and concentration, as expressed in the first normal stress difference and the transient elongational viscosity) and processing conditions (temperature, throughput, draw ratio, capillary dimensions and cooling conditions).

In Section 4 finally, the model thus developed will be applied to the vortex size in contraction flows. Considering the main flow in circular contractions as an isothermal spinning process, the draw ratio equals the imposed contraction ratio squared. The strain rate depends on this draw ratio and the distance between the contraction and the detachment point, i.e. the vortex size. This analogy allows for exactly the same analysis as is used a little further downstream in the spinning line, provided that the shear stress at the vortex boundary is neglected with respect to the elongational stress in the main flow.

\section{Modelling of spinning, a survey}

\subsection{Isothermal spinning of Newtonian fluids}

The essence of the treatment will be illustrated with the most simplified model. Neglecting inertia, gravity and drag forces in a one-dimensional isothermal flow, the continuity equation reads

$Q=A_{0} v_{0}=A_{z} v_{z}$ 
The momentum equation, in terms of the total stress $\sigma_{z z}$, reduces to $\sigma_{z z}=F / A_{z}$.

Further, the constitutive equation for a Newtonian fluid yields

$\sigma_{z z}=\eta_{\mathrm{e}} \frac{\mathrm{d} v_{z}}{\mathrm{~d} z}$.

Solution of eqns. (1)-(3) gives the velocity profile in terms of the spinning force:

$v_{z}=v_{0} \exp \left(\frac{F z}{Q \eta_{\mathrm{e}}}\right)$

or, if a second boundary condition, $v=v_{1}$ at $z=L$, is prescribed, after elimination of the spinning force we have

$\frac{v_{z}}{v_{0}}=\left(\frac{v_{1}}{v_{0}}\right)^{z / L}$ and $F=\frac{Q \eta_{\mathrm{e}}}{L} \ln \left(\frac{v_{1}}{v_{0}}\right)$.

A concave velocity profile is found.

\subsection{Non-isothermal spinning of Newtonian fluids}

It is instructive to investigate the change of the velocity profile occurring when a temperature profile over the spinning line is prescribed. For instance a linear profile:

$\frac{T-T_{0}}{T_{1}-T_{0}}=\frac{z}{L}$

With an Arrhenius type of temperature dependency of the viscosity:

$\eta_{\mathrm{e}}=\eta_{0} \exp \left[-b\left(T-T_{0}\right)\right]$

a position-dependent viscosity is found:

$\eta_{z}=\eta_{0} \exp \left(c \frac{z}{L}\right)$

with $c=-b\left(T_{1}-T_{0}\right)$, which expresses the value of the viscosity ratio between $z=L$ and $z=0$. Substituting eqn. (8) in eqn. (3) and solving yields

$\frac{v_{z}}{v_{0}}=\left(\frac{v_{1}}{v_{0}}\right)^{\epsilon}$,

with

$\epsilon=\frac{\exp (-c z / L)-1}{\exp (-c)-1}$ 


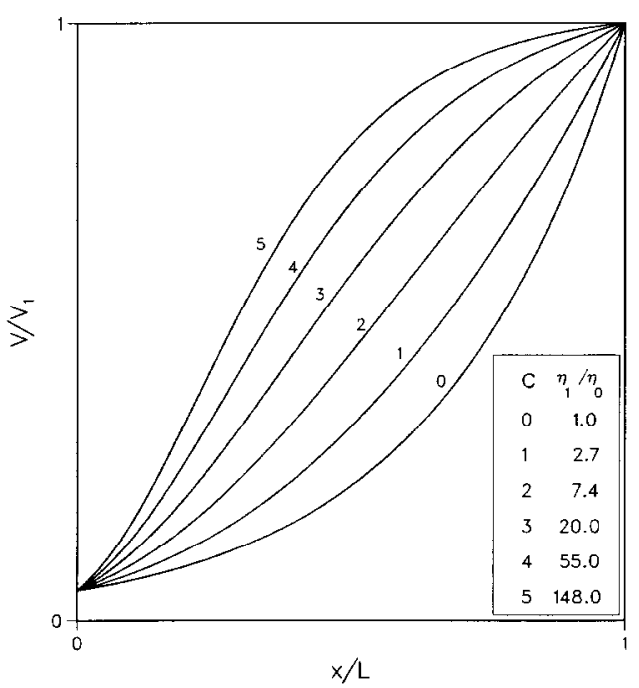

Fig. 1. Velocity profiles in isothermal spinning. The parameter $c$ expresses the viscosity ratio between $x=0$ and $x=L$.

Now convex velocity profiles are found, see Fig. 1. Of course, a linear temperature profile over the spinning line is not very realistic, but also non-linear prescribed profiles:

$$
\frac{T-T_{0}}{T_{1}-T_{0}}=\left(\frac{z}{L}\right)^{\alpha}
$$

still yield an analytical result ${ }^{1}$ (eqn. (9)) with now

$\epsilon=\frac{z}{L}+\sum_{n=1}^{\infty} \frac{(-1)^{n}\left(\frac{z}{L}\right)^{n \alpha+1}}{n !(n \alpha+1)} / 1+\sum_{n=1}^{\infty} n !(n \alpha+1)$.

However, this result is not very useful because the numerical solution of the coupled energy and momentum equations is more realistic and hardly more difficult than the evaluation of eqns. (9) and (12).

\subsection{Isothermal spinning of a viscoelastic fluid}

Numerous studies have been devoted to the spinning of viscoelastic fluids using different constitutive equations, see for example Refs. 5 and 8. Our goal here is to arrive at a simplified approach, which still accounts for the

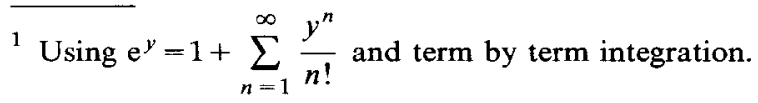


viscoelasticity of the fluid. The constitutive equation for a generalized Maxwell model reads [5]

$\boldsymbol{\sigma}+\lambda\left[\stackrel{\circ}{\boldsymbol{\sigma}}-a\left(\boldsymbol{L} \cdot \boldsymbol{\sigma}+\boldsymbol{\sigma} \cdot \boldsymbol{L}^{T}\right)\right]=2 \eta \boldsymbol{D}$,

where $a$ can be interpreted as a kind of 'molecular slip parameter', resulting in affine or non-affine deformation [31]:

$a=-1$ : lower convected Maxwell,

$a=0$ : corotational Maxwell,

$a=1$ : upper convected Maxwell.

Simple elongational flows are characterized by symmetry and absence of vorticity, we have

$\boldsymbol{L}=\boldsymbol{L}^{T}+\boldsymbol{D}=\nabla \boldsymbol{v} \quad$ and $\dot{\boldsymbol{\sigma}}=\dot{\boldsymbol{\sigma}}$.

Consequently

$\sigma+\lambda(\dot{\sigma}-2 a \sigma \cdot D)=2 \eta D$,

with $D$ being the rate of strain tensor in the spinning process:

$\boldsymbol{D}=\left[\begin{array}{ccc}\frac{\mathrm{d} v}{\mathrm{~d} z} & 0 & 0 \\ 0 & -\frac{1}{2} \frac{\mathrm{d} v}{\mathrm{~d} z} & 0 \\ 0 & 0 & -\frac{1}{2} \frac{\mathrm{d} v}{\mathrm{~d} z}\end{array}\right]$.

The momentum equations in the 1 and 2 directions ( $z$ and $r$ directions, respectively) in terms of the extra stress yield

$\sigma_{11}(z)-p=\frac{F}{A_{z}}, \quad \sigma_{22}(z)-p=0$.

Upon eliminating the unknown isotropic pressure, $p$, the following equation is obtained:

$\sigma_{11}(z)-\sigma_{22}(z)=\frac{F}{A_{z}} \stackrel{(1)}{=} \frac{F}{Q} v$.

For a steady-state spinning process, the combination of eqns. (15), (16) and (18), with $\dot{v}=v \frac{\mathrm{d} v}{\mathrm{~d} z}$, yields

$v+\lambda\left\{v \frac{\mathrm{d} v}{\mathrm{~d} z}-\frac{Q}{F} a\left[2 \sigma_{11}(z)+\sigma_{22}(z)\right]\right\}=\frac{3 Q \eta}{F} \frac{\mathrm{d} v}{\mathrm{~d} z}$,

or, in combination with eqn. (18)

$v+\lambda\left[(a+1) v \frac{\mathrm{d} v}{\mathrm{~d} z}-3 a \frac{\mathrm{d} v}{\mathrm{~d} z} \sigma_{11}(z) \frac{Q}{F}\right]=\frac{3 Q \eta}{F} \frac{\mathrm{d} v}{\mathrm{~d} z}$. 
This equation can be solved in the standard manner by eliminating the unknown $\sigma_{11}(z)$ by differentiating eqn. (20), see Ref. 32. However, if $\sigma_{22}(z)$ is neglected in eqn. (19) with respect to $\sigma_{11}(z)$, then in this stage of the derivation the approximation:

$\sigma_{11} \approx \frac{F}{Q} v$

can be used (c.f. eqn. (18)), and an analytical expression results [33]:

$v+\lambda(1-2 a) v \frac{\mathrm{d} v}{\mathrm{~d} z}=\frac{3 Q \eta}{F} \frac{\mathrm{d} v}{\mathrm{~d} z}$.

This expression can be integrated, with the boundary condition $v=v_{0}$ at $z=0$ to give

$z=\frac{3 Q \eta}{F} \ln \left(\frac{v}{v_{0}}\right)-\lambda(1-2 a)\left(v-v_{0}\right)$.

This is a simple result. Immediately the viscous solution eqn. (4) is recognized and viscoelasticity apparently only yields an extra term. The ability to distinguish between the relaxation time $\lambda$ and the slip parameter $a$ is lost, however. Note that for the corotational model $(a=0)$ the solution (eqn. (23)) is exact (see Petrie [34]), which means that familiar limitations are to be expected [35]. The approximation expressed in eqn. (21) is the same as that used by Zeichner [36], and yields similar results, see also Ref. 7 .

If the second boundary condition is given in terms of velocity, $v=v_{1}$ at $z=L$, then the spinning force $F$ can be found directly from eqn. (23), compare eqn. (5) with $\eta_{\mathrm{e}}=3 \eta$ :

$F=\frac{3 Q \eta \ln \left(v_{1} / v_{0}\right)}{L+\lambda(1-2 a)\left(v_{1}-v_{0}\right)}$.

The straightening of the velocity profile is one of the most obvious results of the viscoelastic models with $0.5<a \leq 1$, see Fig. 2 .

A number of simulations show that the analytical approximation (eqns. (23) and (24)) compares remarkably well with the complete numerical solution (eqn. (20)), at least for values of $a \geq 0.5$. Apparently, the approximation $2 \sigma_{11} \gg \sigma_{22}$ in eqn. (19) is possible for these values of $a$. It is, however, not yet clear how general this simplification is. For example, values of $a<0.5$ can result in a change of sign of the strain rate. For $a=0$ this happens when $3 Q \eta / F v-\lambda=0$ (see Petrie [34]). These unrealistic changes in sign are found in both analytical and numerical simulations. For further discussion, see Ref. 37.

Comparing eqns. (23) and (4) it is clear that a value of $a=0.5$ yields the Newtonian solution. Also here the question arises how general this result is, 


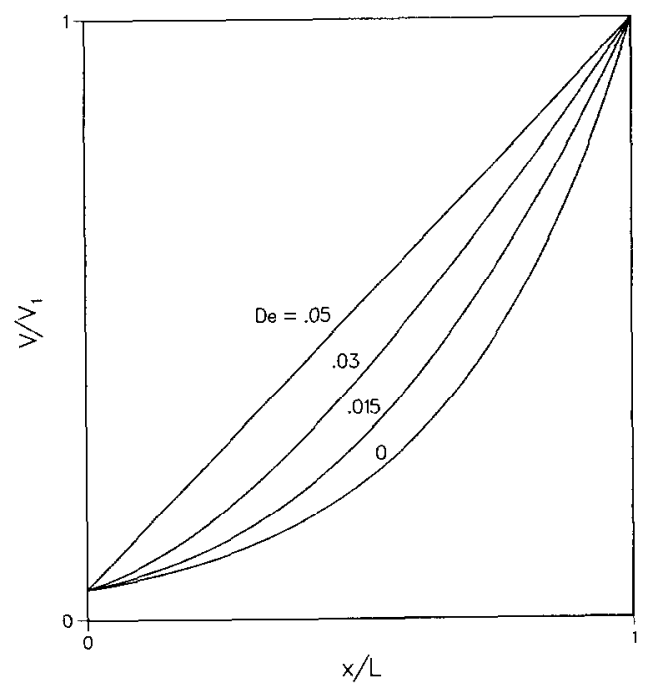

Fig. 2. Velocity profiles for isothermal spinning of a viscoelastic fluid (generalized Maxwell model). The parameter used is the Deborah number $D e=\lambda v_{0} / L(0 \leq D e \leq 0.05)$.

at least for steady extensional flows. Consequently, from eqn. (15):

$\dot{\sigma}-\sigma \cdot(\nabla v) \stackrel{?}{=} 0$

or, with $\frac{\partial \sigma}{\partial t}=0$,

$(v \cdot \nabla) \boldsymbol{\sigma} \stackrel{?}{=} \sigma \cdot(\nabla v)$.

In general, this is true only in a spinning process where $\sigma_{22}$ is neglected with respect to $\sigma_{11}$, see Appendix A.

2.4. Non-isothermal spinning of a viscoelastic solution with evaporating solvent

This problem was solved a number of years ago by Ohzawa and Nagano $[38,39]$ for a Newtonian fluid with a concentration- and temperature-dependent viscosity. Using their analysis, temperature, concentration and velocity profiles are predicted for a given spinning force, see Fig. 3, which is based on data which are representative of the gelspinning process, see below. The strong dependence of the stress-strain curves (spinning force versus draw ratio) on the solvent vapour fraction in the quench air is demonstrated in Fig. 4. Introducing a viscoelastic constitutive equation into Ohzawa and Nagano's system in straightforward now, especially if the simplification, eqns. (21) and (22), is used. Then, instead of eqn. (20), eqn. (22),

$\frac{\mathrm{d} v}{\mathrm{~d} z}=\left[\frac{3 Q \eta}{F v}-\lambda(1-2 a)\right]^{-1}$ 


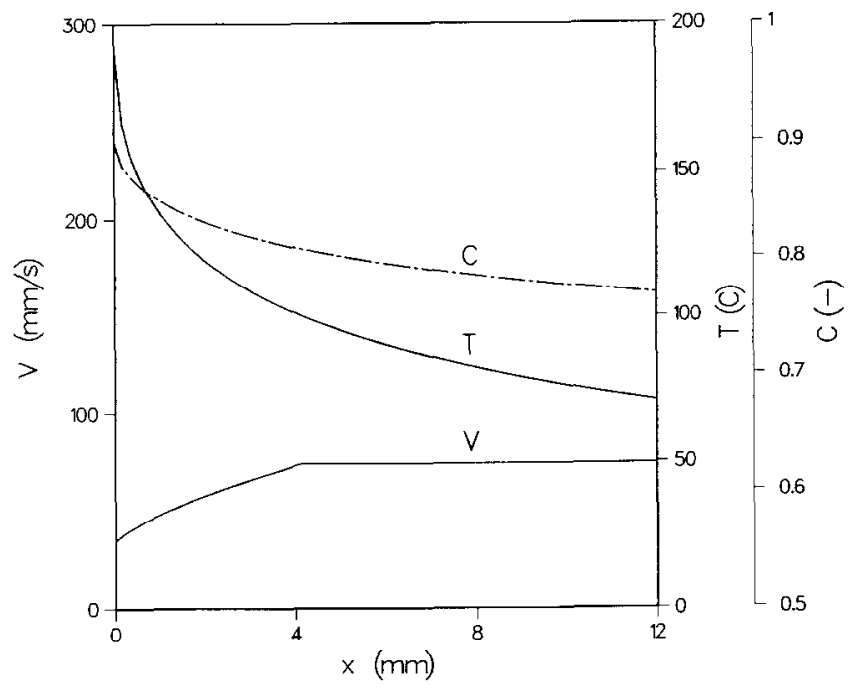

Fig. 3. Calculated velocity (V), temperature (T) and concentration (C) profiles for spinning of a Newtonian solution with evaporating solvent. Data used: $F=15 \mathrm{cN}, c_{0}=0.9, v_{0}=0.03 \mathrm{~m}$ $\mathrm{s}^{-1}, T_{0}=190^{\circ} \mathrm{C}, T_{\text {air }}=45^{\circ} \mathrm{C}, T_{\mathrm{c}}=100^{\circ} \mathrm{C}, c_{\mathrm{p}}=2400 \mathrm{~J} \mathrm{~kg}^{-1}{ }^{\circ} \mathrm{C}^{-1}, b=0.017, \eta_{0}=7000 \mathrm{~Pa}$ $\mathrm{s}, x_{\infty}=0, D_{0}=1 \mathrm{~mm}, k=7.5 \mathrm{~h}$.

has to be solved, with the expressions for the flow rate and viscosity as given in Refs. 38 and 39.

Simulations show that, in the gelspinning process, the velocity and temperature profiles are hardly influenced by the introduction of viscoelasticity.

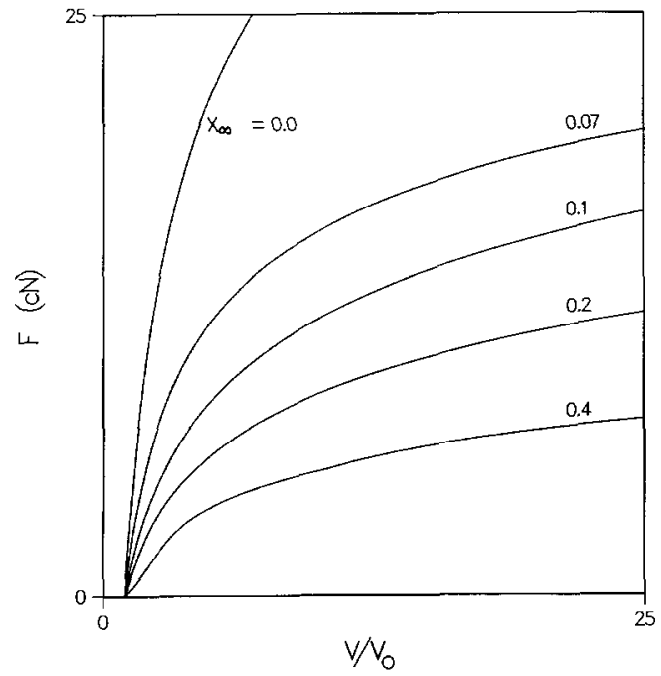

Fig. 4. Calculated spinning force versus draw ratio as a function of the solvent vapour fraction $x_{\infty}$ in the quench air. Data same as for Fig. 3, with $T_{0}=170^{\circ} \mathrm{C}, T_{\mathrm{c}}=110^{\circ} \mathrm{C}$. 


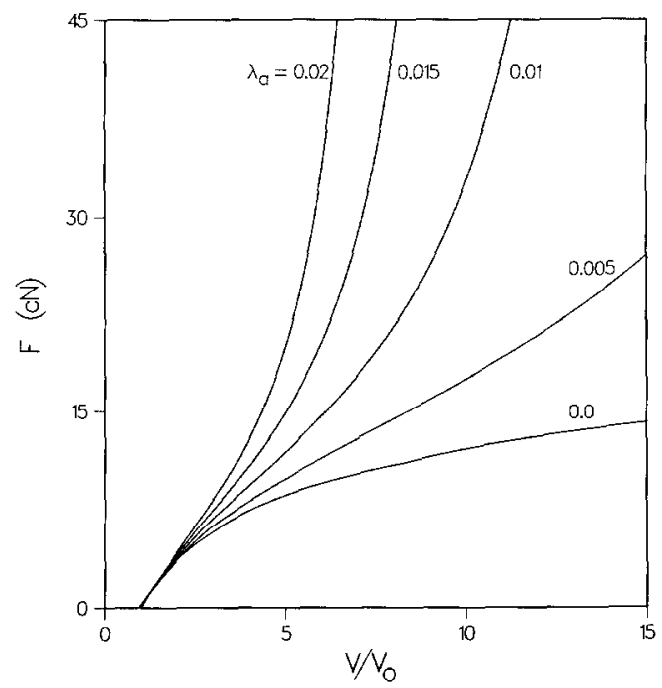

Fig. 5. Influence of viscoelasticity (as expressed in the relaxation time $\lambda_{a}=\lambda(1-2 a)$ ) on the relation between spinning force and draw ratio. Data same as for Fig. 4 with $x_{\infty}=0.1$.

The reason is that boundary conditions are given in terms of prescribed velocities and that the problem is dominated by fast cooling due to evaporation of solvent. However, viscoelasticity has a pronounced influence on the spinning force-draw ratio relations, see Fig. 5.

\section{Pullout}

\subsection{Qualitative description of pullout}

The analysis so far provides the necessary tools for solving the main solution spinning problem of concern here: the pullout phenomenon and a quantitative description thereof.

The solution is pulled out of the capillary at the usual draw ratios. This is illustrated in Fig. 6. The solution consists of 15\% UHMWPE (Himont HB $312, \mathrm{Mw} 1500 \mathrm{~kg} \mathrm{~mol}^{-1}$ ) in decalin. The solution temperature is $180^{\circ} \mathrm{C}$. The capillary diameter is $1 \mathrm{~mm}$ and in this case the throughput is $21 \times 10^{-9} \mathrm{~m}^{3}$ $\mathrm{s}^{-1}$. Factors promoting pullout are revealed in Fig. 7 which shows the stress-strain curves (spinning force versus draw ratio) for two vapour concentrations in the ambient atmosphere of the filament: $0 \%$ and $30 \%$. The extreme cooling due to evaporation of the solvent results in a steep line, as discussed before. However, the rate of evaporation is rather sensitive to the vapour concentration in the atmosphere. With increasing vapour concentration in the surrounding air, the slope of the line is substantially decreased, 


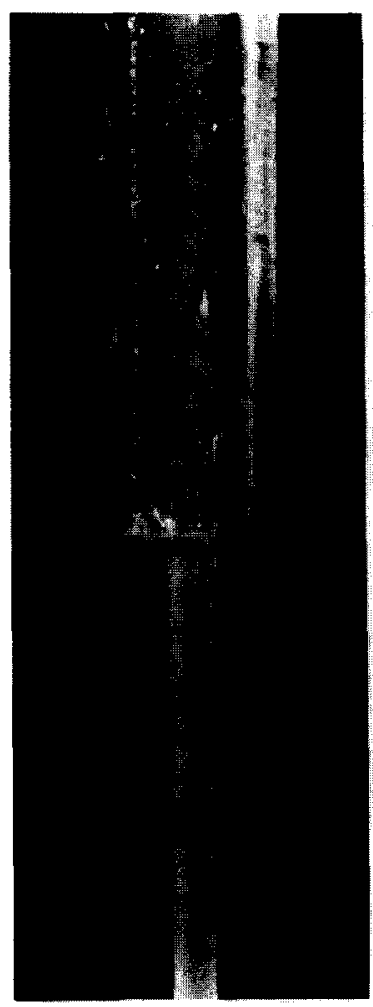

a

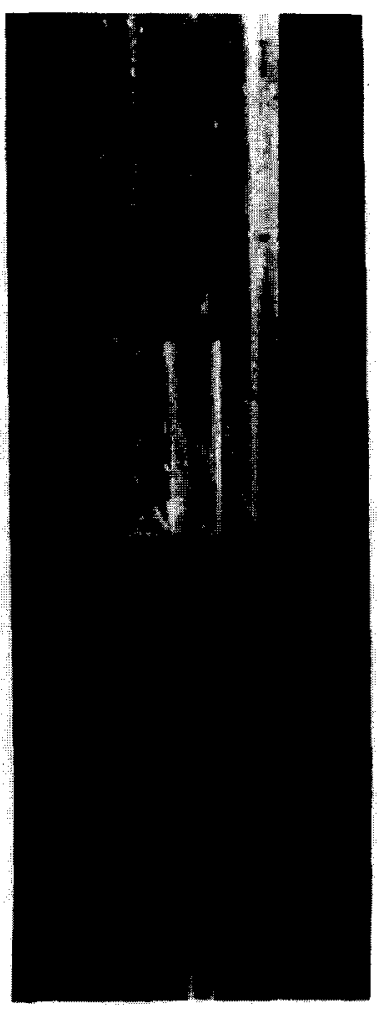

b

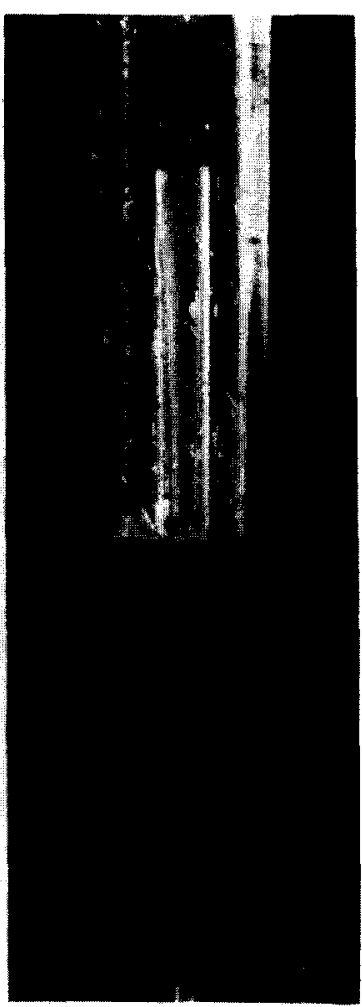

C

Fig. 6. Pullout: the draw ratio increases from (a) to (c).

tending towards an 'isothermal' drawing behaviour. When pullout occurs, stretching takes place inside and outside the capillary. Inside the capillary the atmosphere is saturated with solvent, so more or less isothermal conditions exist, while outside the die the evaporation of solvent is promoted by the vapour-free quench air. As can be concluded from a horizontal line in Fig. 7, at a given spinning force most of the stretching will takc place inside the die. This is confirmed by laser Doppler velocity measurements outside the die. An example of the results is given in Fig. 8. The theoretical velocity profiles inside and outside the die are compared with some experimental points. In the calculations the pullout length is prescribed, as well as the average velocity $v_{0}$ in the capillary and the end velocity $v_{1}$; approximated material data are used. As indicated above, both models, viscous and viscoelastic, give roughly the same result, underlining the fact that the velocity profiles are dominated by the thermal boundary conditions, or, more precisely, by the rate of evaporation. 


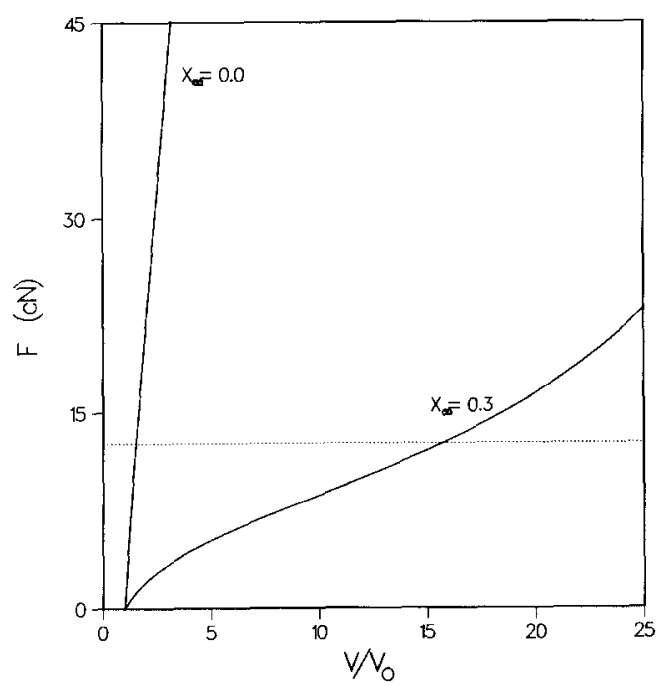

Fig. 7. Spinning force versus draw ratio for two solvent vapour fractions in the quench air. The horizontal line shows the strong difference in draw ratio at a constant spinning force. Data same as for Fig. 4 with $\lambda_{a}=5 \times 10^{-3} \mathrm{~s}^{-1}$.

A number of questions now can be asked: what is the prerequisite for pullout, when does pullout start, what is the position of the detachment point, for example in dependence of $v_{0}$ and $v_{1} / v_{0}$, and is it possible to develop a quantitative model? Simulations with 'Polyflow' [40] revealed that

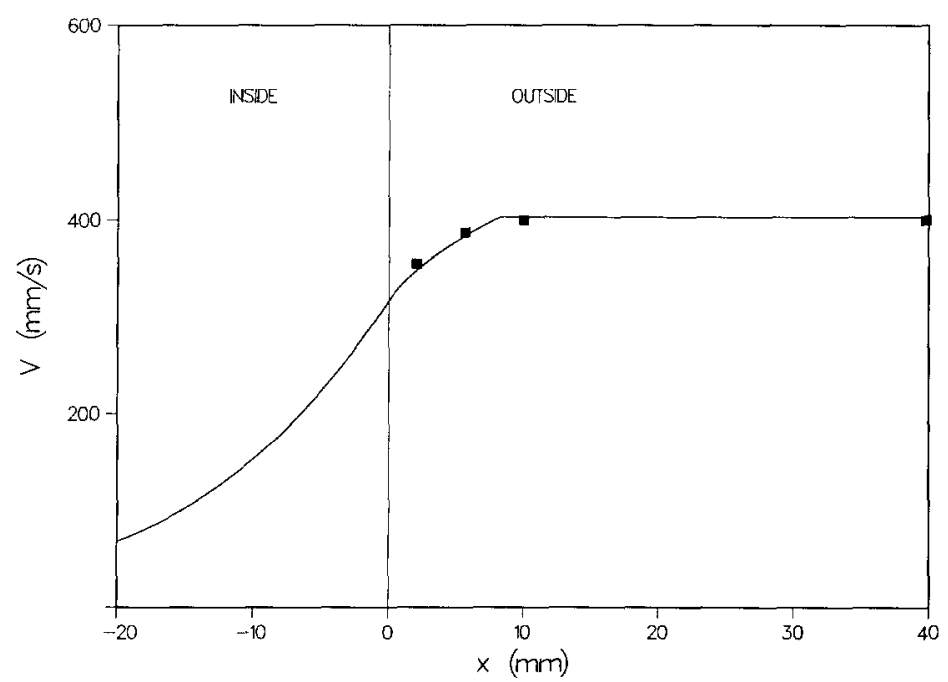

Fig. 8. Measured and calculated velocity profiles inside and outside the die. Data same as for Fig. 4 with $v_{0}=0.06 \mathrm{~m} \mathrm{~s}^{-1}, F=3.3 \mathrm{cN}, \lambda_{a}=0.01 \mathrm{~s}^{-1}, c_{0}=0.85$. 
in standard melt spinning, pullout is very unlikely to occur. The spinning force necessary to compensate for extrudate swell is so high that in a real experiment the melt strength will be exceeded and the filament will break. The solutions used in our experiments not only possess an extremely high 'melt' strength (the value of which strongly depends on the degree of orientation of the molecules in the solution and can range from $0.5 \mathrm{MPa}$ for isotropic solutions to over $10 \mathrm{MPa}$ for highly oriented solutions) but are also strongly shear thinning (power law index $n \approx 0.16$ ) and exhibit wall slippage. Nevertheless, the analysis will be of interest for normal melts as well, as will be discussed below. Besides, in model liquids, such as the Boger fluids, pullout also occurs [29]. Sridhar's results [29] will be discussed below.

Focussing the attention on the detachment point, where the solution leaves the wall, pullout can be analysed. This statement originates from the fact that the stress at this point will be independent of the position of the detachment point inside the capillary (compare Figs. 6(b) and 6(c)). This implies that the spinning force should be constant once pullout has started, as long as entrance effects do not appear. With the experimental set up shown in Fig. 6 this could not be verified. More careful experiments with a new (long) glass die ( $D=1 \mathrm{~mm} ; L / D=60$ ) surrounded by thermostatic oil, in order to maintain isothermal conditions over the die, gave much better results, see Fig. 9.

The measured spinning force $F$, the pullout length $l_{\text {po }}$ and the total pressure drop over the die are given as a function of the draw ratio, keeping the throughput constant. The spinning force follows the theory for non-isothermal spinning (see Fig. 7) until pullout starts at a critical value $F_{\mathrm{c}}$ (Fig. 9(a)). After that point the force remains constant until it starts to increase again at higher draw ratios. If $F=F_{c}$ the pullout length starts to increase with increasing draw ratio (see Fig. $9(\mathrm{~b})$ ). This is also reflected in the decrease in the total pressure drop over the capillary $\Delta P$. The pullout length can be calculated from the change in pressure drop, even in metal dies, because this change reflects the effective shortening of the capillary.

\subsection{A quantitative model for pullout}

The position of the detachment point and the stability of this point can be understood from a stress balance at this point. The position is set by balancing the force necessary to stretch the filament (spinning force) and the stress that the fluid exerts normal to the wall due to its deformation history. The total normal stress due to the spinning force, $\sigma_{z z}$, and the normal stress at the wall, $\sigma_{r r}$, are connected through the first normal stress difference $N_{1}$ :

$N_{1}=\sigma_{z z}-\sigma_{r r}$ 

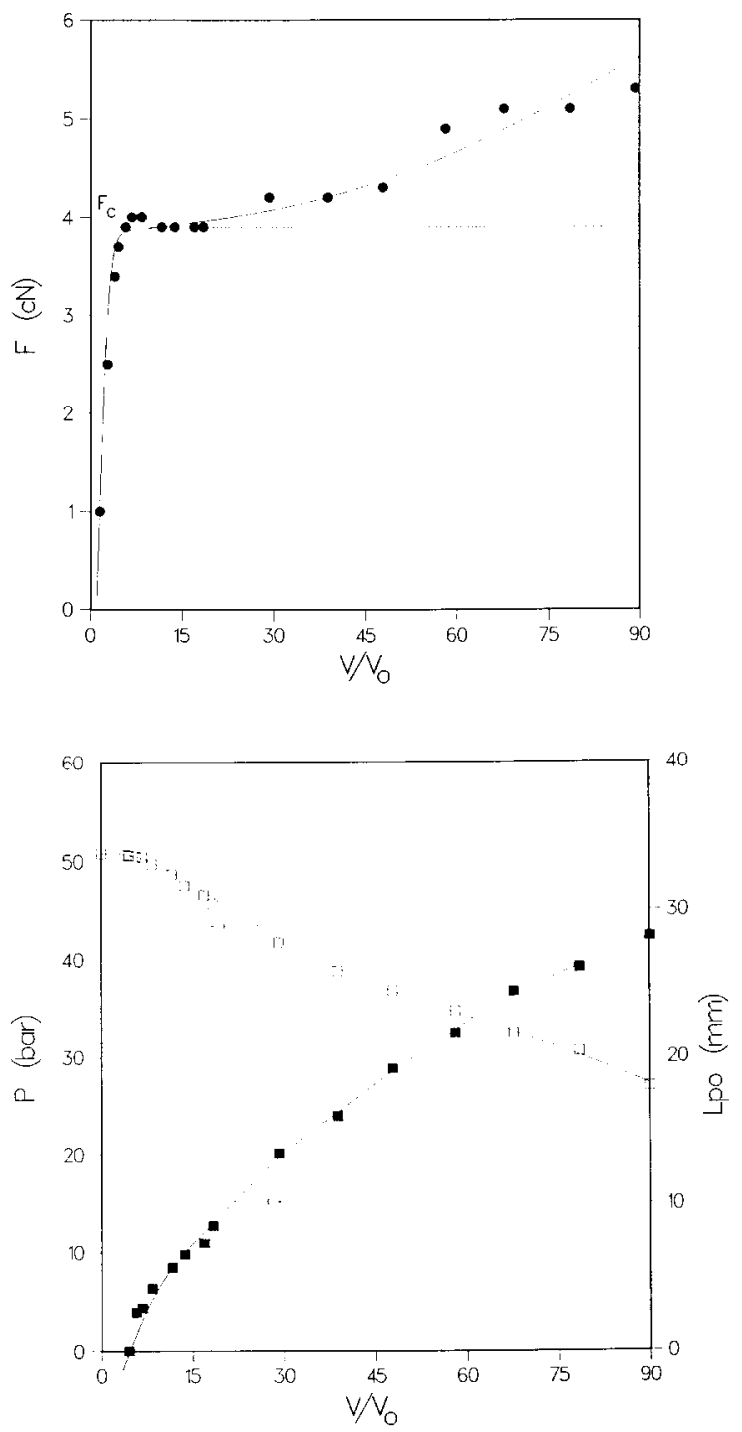

Fig. 9. (a) Experimental spinning force versus draw ratio for a capillary of $1 \mathrm{~mm}$ diameter and a length of $60 \mathrm{~mm}$ after the contraction. After the plateau value $F_{\mathrm{c}}$ at the start of pullout (see part (b)) the increase in the spinning force reflects the relaxing extra stress, built up in the upstream contraction (see Fig. 10) For data see text. (b) Measured pullout length versus draw ratio ( $\square$ ) and total pressure drop over the die ( $\square$ ).

In a first approximation the spinning tension at the detachment point can be set equal to the average total normal stress in the $z$ direction:

$\sigma_{z z}=\frac{F}{\pi R^{2}}$. 
Furthermore, at the detachment point, the total normal stress exerted on the wall is zero, in order to allow the fluid to leave the wall, i.e.

$\sigma_{r,}=0$.

(Surface tension is neglected here.) Combining eqns. (28), (29) and (30) gives a relation between the spinning force, $F$, and the normal stress difference, $N_{1}$, at the detachment point:

$\frac{F}{\pi R^{2}}=N_{1}$.

This yields the critical force, $F_{c}$, at which pullout starts (see Fig. 9):

$F_{\mathrm{c}}=N_{1} \pi R^{2}$.

A more accurate stress balance at the detachment point can be obtained from an integral momentum balance, analogous to Refs. 41 and 42 , including the spinning force (see Appendix B):

$F_{c}=2 \pi \int_{0}^{R}\left(N_{1}+\frac{N_{2}}{2}\right) r \mathrm{~d} r$,

with $N_{2}$ the second normal stress difference, which is much smaller than the first ( $N_{2} \approx-0.1 N_{1}$ ). Equation (33) can be interpreted as $F_{\mathrm{c}}=\bar{N}_{1} \pi R^{2}$, where $\bar{N}_{1}$ is the normal stress difference averaged over the cross-section of the capillary and equals the extra stress at the wall (i.e. the total stress minus the isotropic pressure) when no spinning force is applied. The effect of the rearrangement of the velocity profile upstream of the detachment point is neglected here.

Calculation of the position of the detachment point for any imposed $v_{0}$ and $v_{1} / v_{0}$ is now straightforward.

In an infinite capillary $\bar{N}_{1}$ depends only on the shear rate distribution. For finite capillaries an extra transient stress must be added, owing to the contraction, see Fig. 10. This transient stress can be calculated with finite element codes, such as Polyflow [40], or can be estimated, see Ref. 13. The development of the normal stress at the wall for a flow through a finite capillary with a contraction is illustrated in Fig. 11(a). Figure 11(b) shows the influence of the shear rate (defined by the average velocity $v_{0}$ ) on $\bar{N}_{1}$ profiles.

However, the force $F$ necessary to stretch the filament from $v_{0}$ to $v_{1}$ in a combined isothermal (inside the die) and non-isothermal (outside the die) spinning process can be calculated with the models described above in section 2, see Fig. 12. For an imposed $v_{0}$ and draw ratio $v_{1} / v_{0}$, the spinning force depends on the isothermal stretching length (i.e. pullout length), see the vertical intersection in Fig. 12. Consequently the spinning stress $F / \pi R^{2}$ 


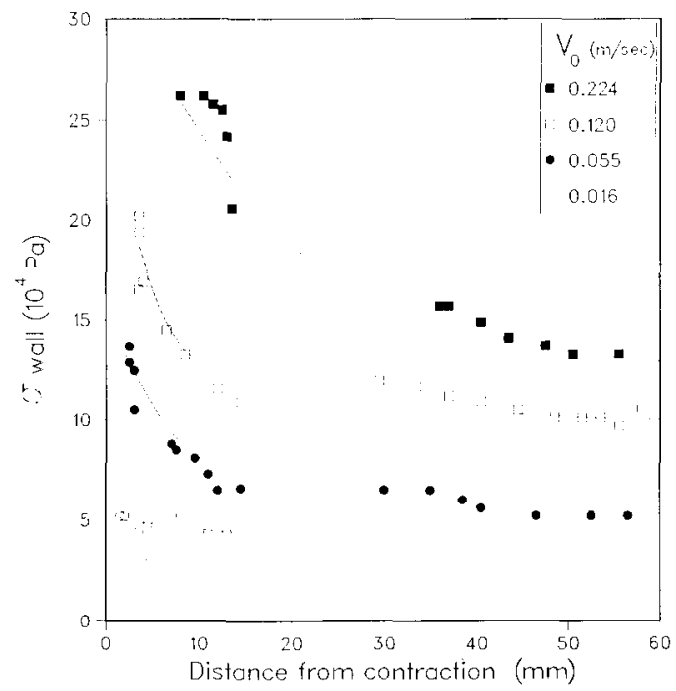

Fig. 10. Relaxation of the stress at the wall after a $8: 1$ contraction with an entrance angle of $60^{\circ}$, determined by measuring the spinning force $F_{c}$ at different throughputs. The capillary length was varied by repeatedly (four times in total) cutting the same die. Data as in Fig. 9.

depends on the position of the detachment point, see Fig. 11. According to eqn. (32) this position is determined by the intersection of the line of the normal stress at the wall $\bar{N}_{1}$ and the spinning stress. At the detachment point $F=F_{\text {c }}$, the force which is prerequisite to start and maintain pullout (eqn. (32) or (33)). The angle of intersection between the two lines reflects the sensitivity of the position of the detachment point to temperature or concentration variations.

Increasing the draw ratio shifts the detachment point in the upstream direction, see Fig. 11(a), compare with Fig. 9. The dependence on the throughput is given in Fig. 11(b). It is interesting to note that if the draw ratio is increased to such an extent that the end of the contraction is reached, no more points of intersection are found. Consequently the pullout length will not cease to increase. This fact could be verified with experiments: as soon as the detachment point has reached the contraction, it disappears into the upstream die, until the filament breaks.

The analysis so far might provide a new method of measuring the first normal stress differences of solutions at high shear rates. The accuracy should not be too poor, because only a stress balance is used and the force is directly recorded. Moreover, only the plateau region of constant force $F_{\mathrm{c}}$ is relevant, provided that the capillary is long enough to eliminate entrance effects, see Appendix C. Finally, it can be concluded that the occurrence of pullout and the position of the detachment point are controlled by a 

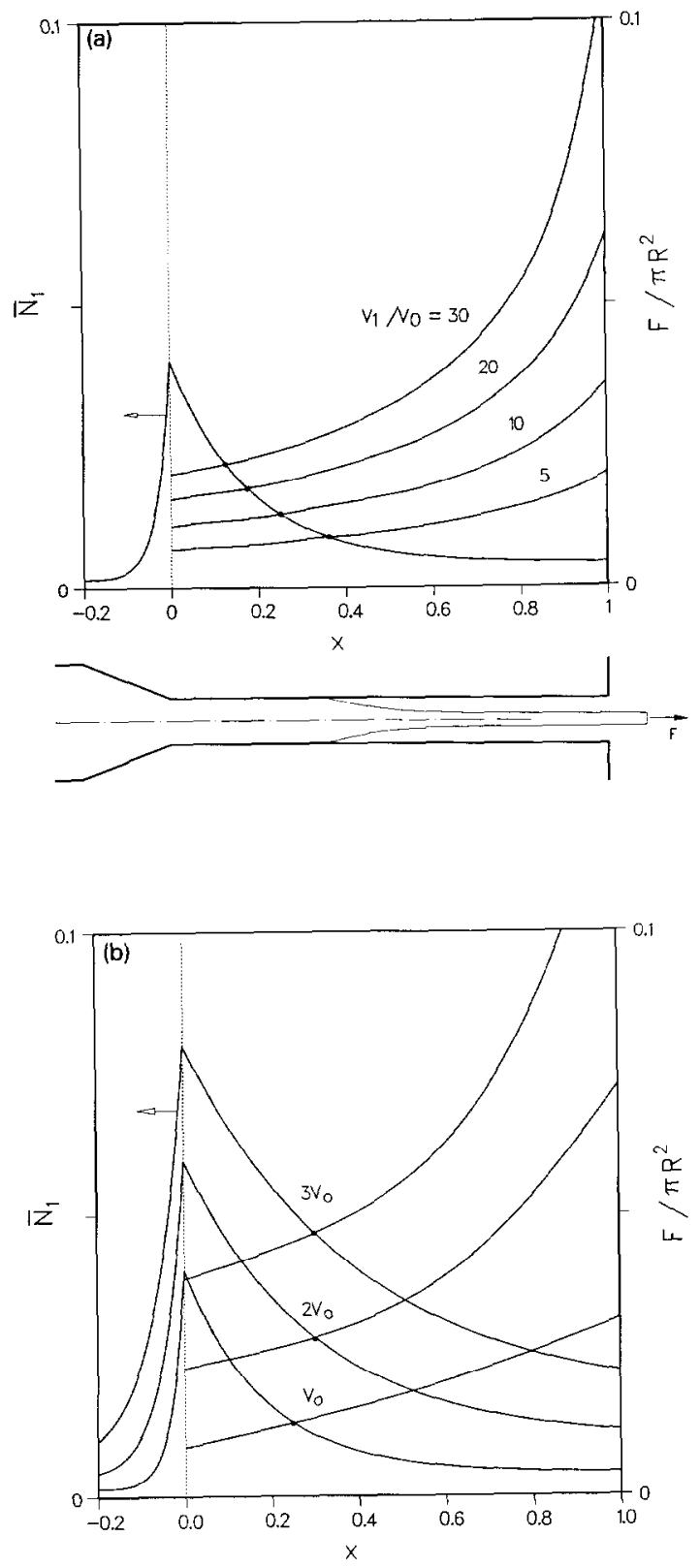

Fig. 11. (a) Development of the averaged normal stress $\bar{N}_{1}$ versus the distance $x$ from the contraction (qualitatively) and the spinning tension as a function of the isothermal stretch length $(1-x)$ with draw ratio. The intersection of both lines gives the position of the detachment point. (b) As for part (a); an increase in throughput at a constant draw ratio influences both lines. The shift of the detachment point now depends on more detailed rheology. 


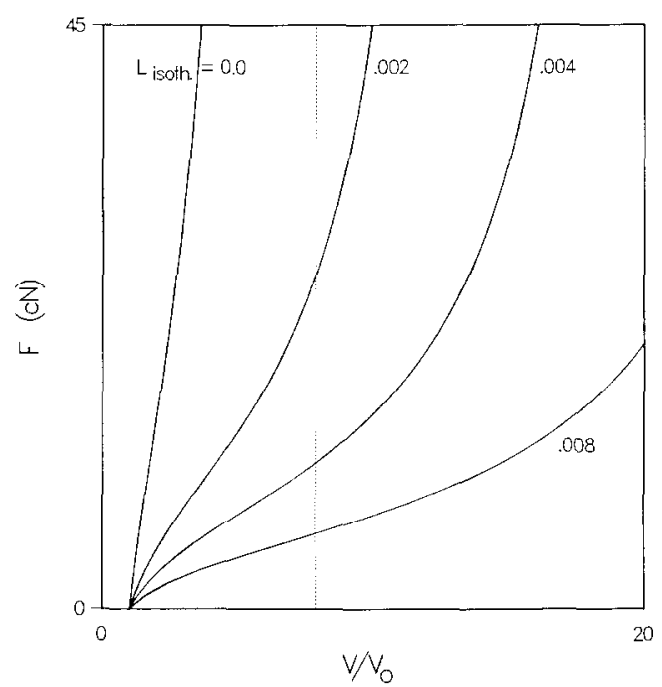

Fig. 12. Calculated force versus draw ratio for different values of the pullout length ( $\left.L_{\text {isothermal }}(\mathrm{m})\right)$.

combination of both the shear rate dependent first normal stress difference $N_{1}(\dot{\gamma})$ and the spinning force $F$ which reflects the transient elongational viscosity $\eta_{\mathrm{e}}$.

\subsection{Evaluation of the model for pullout}

\subsubsection{Basic equations}

First normal stress differences and shear viscosities can often be approximated by power law relations:

$N_{1}=\psi_{0} \dot{\gamma}^{m}$,

$\eta=\eta_{0} \dot{\gamma}^{n-1}$.

In a tube flow the shear rate as a function of the radius then reads

$\dot{\gamma}=\frac{3 n+1}{4 n}\left(\begin{array}{c}r \\ R\end{array}\right)^{1 / n} \dot{\gamma}_{\text {app }}$,

with $\dot{\gamma}_{\text {app }}$ being the apparent shear rate: $4 Q / \pi R^{3}$. The critical spinning force, $F_{\mathrm{c}}$, can be calculated using these relations and eqn. (33) with $N_{2}=0$ :

$F_{\mathrm{c}}=2 \pi \int_{0}^{R} N_{1} r \mathrm{~d} r$.

Combining eqns. (34)-(37):

$\frac{F_{\mathrm{c}}}{\pi R^{2}}=2 \psi_{0} \frac{n}{m+2 n}\left(\frac{3 n+1}{4 n} \dot{\gamma}_{\mathrm{app}}\right)^{m}$. 
For an upper convected Maxwell model $(a=1)$ with a shear rate dependent viscosity, this equation can be evaluated using

$\psi_{0}=2 \eta_{0} \lambda$ and $m=n+1$.

Finally, with a constant shear viscosity $(n=1)$, the expression for the critical spinning force reduces to

$F_{\mathrm{c}}=16 \pi \lambda \eta v_{0}^{2}$ with $v_{0}=Q / \pi R^{2}$.

\subsubsection{Comparison with Sridhar's experiments}

To our knowledge the only independent data on pullout, allowing for an objective evaluation of the model, originate from Sridhar and Gupta [29]. They measure the critical stress at which pullout starts, dependent on the throughput, using a model liquid (PAA (Separan AP30) in 50\% aqueous glycerol solutions). Their experiments are completed with the rheological data on $N_{1}(\dot{\gamma})$ and $\eta(\dot{\gamma})$. Their rheological data can be summarized using the power law expressions, eqns. (34) and (35), with

$\psi_{0}=18.8 \mathrm{~Pa} \mathrm{~s}^{m+1}, n=0.41, m=0.77$.

At low shear rates $(\dot{\gamma}<1)$ the viscosity is overestimated using eqn. (35), but this will only have a minor influence on the calculation of the velocity profile. Combined with the value of the radius of the capillary they used, $R=10^{-3} \mathrm{~m}$, the critical spinning tension is found from eqn. (38):

$$
\frac{F_{\mathrm{c}}}{\pi R^{2}}=12.3 \dot{\gamma}_{\mathrm{app}}^{0.77} \text {. }
$$

In Fig. 13 the measurements of Ref. 29 are compared with our calculations. The theory underestimates the critical force. This discrepancy can in part be attributed to the effect of the surface tension. If the surface tension is taken into account the agreement between thcory and experiments is slightly improved. The fact that the experimental points give higher critical stresses and that the discrepancy between experiment and model prediction increases with increasing shear rates could indicate that the capillary used by Sridhar and Gupta is too short. In that case a part of the transient stresses due to the contraction also contributes to the critical stress, see Fig. 11. No data on the capillary used could be found in Ref. 29.

\subsubsection{Comparison with solution spinning experiments}

For evaluating our experiments on spinning of solutions of polyethylene in decalin, eqns. (38) and (39) are used, since it proved to be extremely difficult to measure the relevant rheological properties. From capillary 


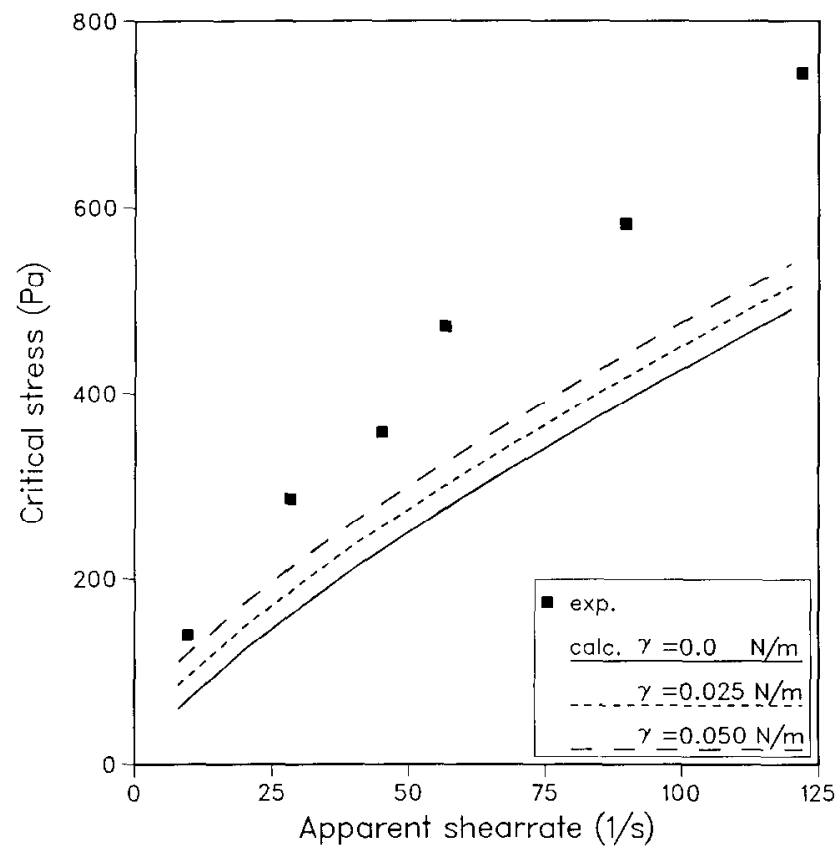

Fig. 13. Critical stress at which pullout starts versus throughput (as expressed in the apparent shear rate). Data from Ref. 29. Model predictions according to eqn. (41) are shown by the drawn line. In the dashed lines two (more or less arbitrary) values of the surface tension $\gamma$ are incorporated.

rheometry the following typical data are found: $Q_{\text {slip }}=0.5 Q_{\text {total }}$ and $\eta_{0}=5500 \mathrm{~Pa} \mathrm{~s}^{n}, n=0.16, \lambda=0.1 \mathrm{~s}$.

For the dic used $\left(R=5 \times 10^{-4} \mathrm{~m}\right)$ and the given throughput $\left(Q=21 \times 10^{-9}\right.$ $\mathrm{m}^{3} \mathrm{~s}^{-1}$ ), the critical spinning force for pullout reads (eqns. (36)-(39))

$F_{\mathrm{c}}=20 \mathrm{cN}$,

reducing to

$F_{\mathrm{c}}=9 \mathrm{cN}$

when a slip factor of 0.5 is included in the flow rate. Notwithstanding the simplicity of the constitutive equation used, these data are of the same order of magnitude as the experimentally determined critical spinning force for our solutions: $F_{\mathrm{c}}$ (experimental) $\approx 4 \mathrm{cN}$ (see Fig. 9).

\section{Vortex size in contraction flows}

An intriguing extension of the analysis developed so far is that the model applies to the inflow problem into a contraction as well, provided that some 


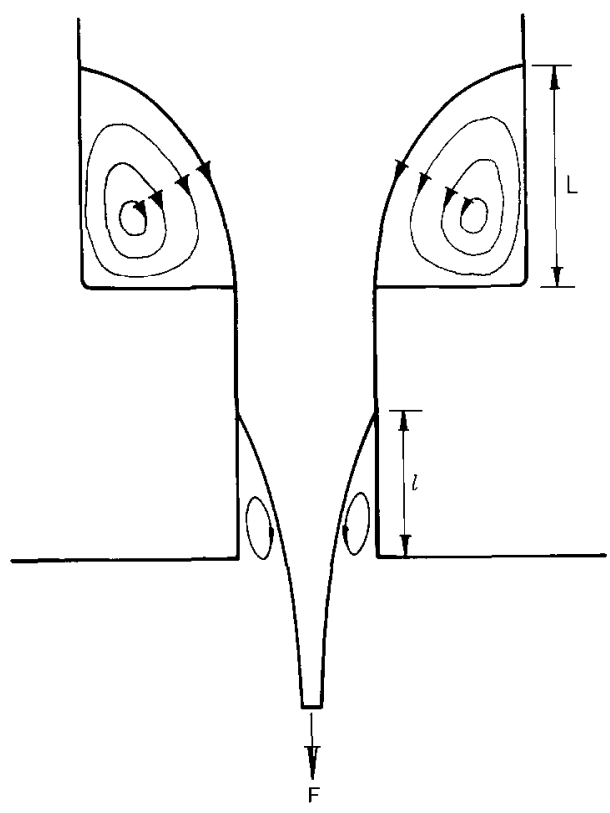

Fig. 14. Analogy between the pullout length in solution spinning and the vortex size in contraction flow.

simplifications are allowed for. In Fig. 14 the analogy is demonstrated. In this respect it is interesting to quote White and Baird [43], who recently reviewed the entry flow problem:

"It is our belief that the origin of vortices is not related to the elasticity based on shear flow properties but to the behaviour of the transient elongational viscosity," and "It is believed that the constitutive equations used in the numerical solution must reflect this extensional behaviour if vortices are to be predicted".

Their statement that the elasticity based on shear properties is no longer important is apparently wrong. It was based on the experimental observations that polyacrylamide solutions and Boger fluids exhibit vortices in axisymmetric flow (with the vortex size increasing with fluid elasticity), but that the vortex is nearly suppressed in planar entry flows, while some polymer melts, such as the 'strain hardening' branched low density polyethylene, exhibit vortices in both planar and axisymmetric flows.

The question whether or not a vortex shows up can be reduced to the prediction of its size: the length $L$ of the vortex, see Fig. 14. This is exactly the same question as that raised in the analysis of pullout: what is the pullout length $l_{\text {po }}$. There are, of course, some minor differences.

(1) In the pullout problem the vortex consists of circulating air, which will hardly exert a shear stress on the accelerating fluid, while in the entry flow 
the solution or melt itself rotates inside the vortex. If, however, the main flow is considered, the shear stress at the boundaries is probably negligible compared with the elongational stress.

(2) In spinning, the shear stresses are neglected and velocities and stresses are taken as constant in each cross-section. The fluid accelerates according to the simple spinning equations, as derived above, while the entry flow is in fact two or even three dimensional; compare for example the experimental data of Raiford et al. [44]. However, again in first approximation, the entry flow can also be modelled as a one-dimensional acceleration problem.

(3) In spinning, the fluid is pulled out of the capillary while being pushed through the contraction. This fact, however, enlarges the scope of the analysis. As already mentioned, only those fluids which combine a high elongational viscosity with pronounced shear thinning (and perhaps also wall slippage is necessary) show pullout. For other polymers or (model) fluids the fibre will break before the critical stress is reached. This limitation is not present in entry flows.

\subsection{Circular contraction}

\subsubsection{Basic equations}

If the attention is focussed on the detachment point of the vortex, where the fluid leaves the wall, then the condition:

$\sigma_{r r}=0$

should be met, setting the 'isotropic' pressure in the vortex to zero. This yields again the criterion:

$$
F_{\mathrm{c}}=2 \pi \int_{0}^{R}\left(N_{1}+\frac{N_{2}}{2}\right) r \mathrm{~d} r .
$$

The only problem which remains is to determine the 'force' $F$ which is necessary to accelerate the fluid. With the simplifications introduced so far, this is an isothermal spinning process with:

$v_{0}=Q / \pi R_{0}^{2}$,

$\frac{v_{1}}{v_{0}}=\left(\frac{R_{0}}{R_{1}}\right)^{2}$

and the stretch length $L$ equal to the vortex size. For this problem the force $F$ is explicitly given in eqn. (5) for the Newtonian case:

$F=\frac{Q \eta_{\mathrm{e}}}{L} \ln \left(\frac{v_{1}}{v_{0}}\right)$ 
However, when a power law relation for the elongational viscosity is used:

$\eta_{\mathrm{e}}=\eta_{\mathrm{e} 0}\left(\frac{\mathrm{d} v_{z}}{\mathrm{~d} z}\right)^{k-1}$

eqn. (5) has to be modified slightly. Combination of eqns. (1), (2) and (47) now yields

$F=\frac{Q \eta_{\mathrm{e} 0}}{L} L^{\alpha}\left[\frac{1}{\alpha}\left(v_{1}^{\alpha}-v_{0}^{\alpha}\right)\right]^{1 /(1-\alpha)}$,

with $\alpha=\frac{k-1}{k}$.

For the transient viscoelastic Maxwell model eqn. (24) can be used:

$F=\frac{3 Q \eta \ln \left(v_{1} / v_{0}\right)}{L+\lambda(1-2 a)\left(v_{1}-v_{0}\right)}$.

The vortex size for different fluids can now be found by a combination of the expression for the critical force (eqn. (43)) and one of the equations for the spinning force (eqns. (46), (48) or (49)). For example, for viscoelastic fluids with a rheological behaviour as described with the power law relations in eqns. (34), (35), and (47) the vortex size results directly from eqns. (43) and (48):

$L=R_{0} \frac{1}{4 \alpha}\left[\left(\frac{R_{0}}{R_{1}}\right)^{2 \alpha}-1\right]\left[\eta_{\mathrm{e} 0} \frac{m+2 n}{2 \psi_{0} n}\left(\frac{4 n}{3 n+1}\right)^{m}\right]^{1-\alpha}\left(\frac{4 Q}{\pi R_{0}^{3}}\right)^{1-m(1-\alpha)}$.

While combination of eqns. (43) and (49) yields the result for the generalized Maxwell fluid:

$L=\left[3 Q \eta \ln \left(\frac{v_{1}}{v_{0}}\right)\right]\left[2 \pi \int_{0}^{R}\left(N_{1}+\frac{N_{2}}{2}\right) r \mathrm{~d} r\right]^{-1}-\lambda(1-2 a)\left(v_{1}-v_{0}\right)$.

For an upper-convected Maxwell model ( $a=1)$, the right-hand side of eqn. (51) can be simplified, using eqns. (33)-(39), while for $n=1$ (constant viscosity), the expression finally reads (eqns. (40) and (51)):

$L=\frac{3 R_{0}^{2}}{16 \lambda v_{0}} \ln \left(\frac{v_{1}}{v_{0}}\right)+\lambda\left(v_{1}-v_{0}\right)$.

Expressing the elasticity in the dimensionless Deborah number

$D e=\frac{\lambda v_{0}}{R_{0}}$

and the geometrical ratio as $\beta$,

$\beta=\frac{R_{0}}{R_{1}}$, 


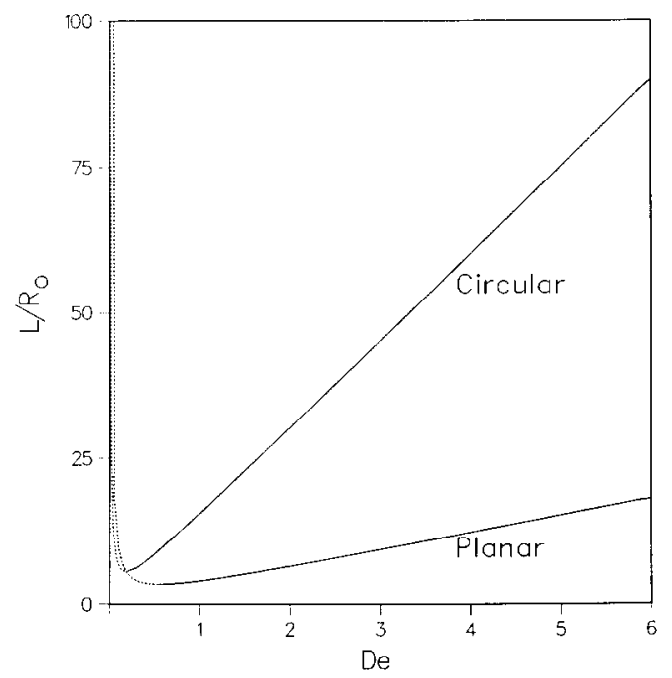

Fig. 15. Vortex size versus Deborah number as calculated with the upper-convected Maxwell model for a circular (eqn. (54)) and a planar (eqn. (62)) 4:1 contraction.

this vortex size can be written as:

$$
\frac{L}{R_{0}}=\frac{3}{8 D e} \ln \beta+D e\left(\beta^{2}-1\right) \text {. }
$$

For the $4: 1$ contraction ( $\beta=4)$, the results are graphically presented in Fig. 15.

Apparently the second (viscoelastic) term in eqn. (54) dominates at the higher Deborah numbers. At extremely low Deborah numbers an irrealistic increase in vortex size is found. This is due to the limitations of the upper-convected Maxwell model while, moreover, in this region the assumptions that shear stresses can be neglected and velocities and stresses taken constant over each cross-section, are almost certainly violated.

\subsubsection{Comparison with Binding's experiments}

It is interesting to compare this analytical result (eqn. (50)) with the results of Binding and Walters [30]. Using a minimum energy approach for the dissipation in the (viscous only) flow from the detachment point into the capillary, they also arrived at an expression for the vortex size. Their prediction of the vortex size as a function of throughput is satisfying, see Figs. 16 and 17 (dotted lines). Since the rheological data of their model fluids ( $1 \%$ and $2 \%$ solutions of PAA in water) were carefully measured, except for, as usual, the transient elongational viscosity, eqn. (50) can be used to predict the vortex size according to the analysis presented in this paper. The constants in eqns. (34) and (35) for viscosity and first normal 


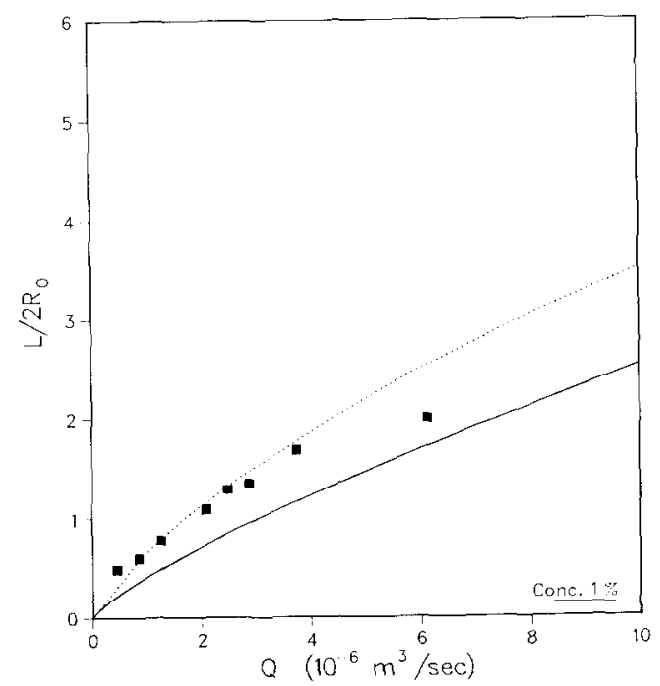

Fig. 16. Vortex size versus throughput for a 1\% aqueous PAA solution. Experimental points from Ref. 30. The dotted line is according to the model of Ref. 30. The solid line shows the prediction according to eqn. (50) with use of the averaged elongational viscosity.

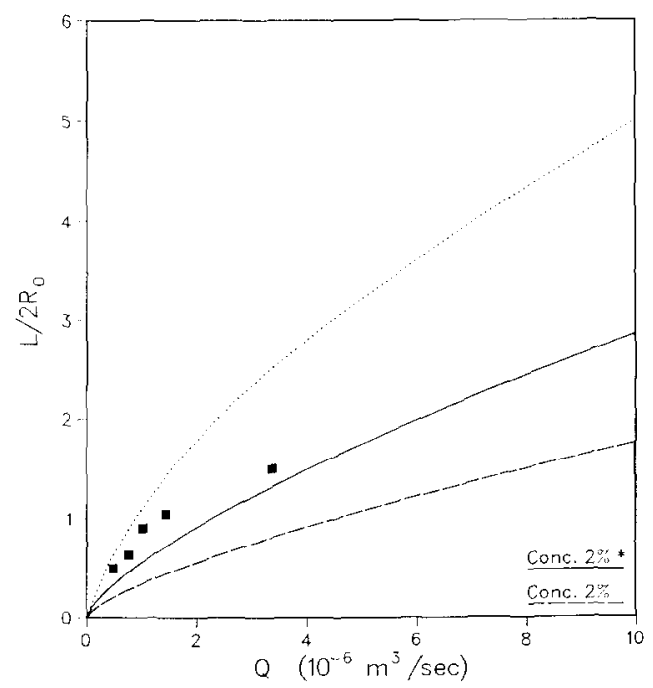

Fig. 17. As Fig. 16, for a $2 \%$ solution. The solid line is based on eqn. (50), using the spinning line data for the elongational viscosity $\left(2 \%^{*}\right)$, while the dashed line in based on an elongational viscosity determined from contraction flow data (2\%). The dotted line is according to the model of Ref. 30 , which used the contraction flow data (2\%). 
stress difference to be used in eqn. (50), as abstracted from Binding and Walters' measurements, read

concentration 1\%: $\eta_{0}=3.16 n=0.34 \psi_{0}=3.71 m=0.81$,

concentration $2 \%: \eta_{0}=20.8 n=0.23 \psi_{0}=100 m=0.6$.

For the elongational viscosity they found (eqn. (47))

concentration $1 \%: \eta_{\mathrm{e} 0}=0.87 k=3.76$,

concentration $2 \%: \eta_{\mathrm{e} 0}=57.3 k-2.28$ (from entry pressure data),

concentration $2 \% * \eta_{\mathrm{e} 0}=174 k=2.28$ (from spin-line rheometer data).

Using these data and the dimensions of the capillary used $\left(R_{0}=3.5 \times 10^{-3}\right.$ $\mathrm{m} ; R_{1}=2.4 \times 10^{-3} \mathrm{~m}$ ), the following relations are obtained, see eqn. $(50)$ :

$\frac{L}{2 R_{0}}=a Q^{b}$

where $Q$ is in cubic centimetres per second, with

\begin{tabular}{lll}
\hline Concentration & $a$ & $b$ \\
\hline $1 \%$ & 0.415 & 0.785 \\
$2 \%$ & 0.342 & 0.71 \\
$2 \% *$ & 0.557 & 0.71 \\
\hline
\end{tabular}

These results are also plotted in Figs. 16 and 17, as solid and dashed lines.

Apparently the averaged elongational viscosity data $(2 \% *)$ as obtained from spin-line rheometer experiments give better predictions (solid line), as could be expected. Also the upswing at low Deborah numbers (low throughputs), which is found for a Maxwell type of constitutive equation (see Fig. 15) does not show up using these, more practical, constitutive relations.

In principle a model based on an energy approach [30] should give similar results to those derived with the model as presented in this paper, which is based on a stress balance. For the $1 \%$ solution (Fig. 16) this seems to be true. However, for the $2 \%$ solution (Fig. 17), larger differences between the two models exist, especially because for the same elongational viscosity (they used the entry pressure data), the dotted line [30] should be compared with our dashed line. For an elongational viscosity, based on spin-line rheometer data $(2 \%$ ), the model of Ref. 30 will yield a line which is even steeper than the one presented (dotted; $2 \%$ ). One obvious difference between the two models can be found at the boundaries between the vortex and the main flow. In Ref. 30, a stick condition to a hypothetical wall is assumed, while in this paper the interaction is considered to be negligible. Also it should be 
noted that viscoelasticity, which is absent in their model, is more easily incorporated in the model derived here, as shown above.

From the results (Figs. 16 and 17), given the conceptual simplicity of the model proposed in this paper, which is based on the application of a direct stress balance at the detachment point, it might be concluded that without any unrealistic assumptions the vortex size can be predicted with reasonable accuracy as a function of fluid properties and process conditions.

\subsection{Planar contraction}

The main difference between an axisymmetric and a planar contraction, with width $W$ and channel height $2 h$, is the acceleration of the fluid as expressed in the geometrical ratio:

$\frac{v_{1}}{v_{0}}=\frac{h_{0}}{h_{1}}=\beta$

for the planar contraction, versus

$\frac{v_{1}}{v_{0}}=\left(\frac{R_{0}}{R_{1}}\right)^{2}=\beta^{2}$

for the circular contraction. For the 'same' contraction ratio $\beta$, the linear versus quadratic acceleration is evident. The rate of strain $\boldsymbol{D}$ for the planar contraction reads

$\boldsymbol{D}=\left[\begin{array}{ccc}\frac{\mathrm{d} v}{\mathrm{~d} z} & 0 & 0 \\ 0 & -\frac{\mathrm{d} v}{\mathrm{~d} z} & 0 \\ 0 & 0 & 0\end{array}\right]$.

For the generalized upper-convected Maxwell model (eqn. (15)), the velocity profile can be obtained, using eqn. (57),

$v+\lambda(1-2 a) v \frac{\mathrm{d} v}{\mathrm{~d} z}=\frac{4 Q \eta}{F} \frac{\mathrm{d} v}{\mathrm{~d} z}$,

following the same derivation as used with eqns. (16)-(23). Integration of eqn. (58) yields

$F=\frac{4 Q \eta \ln \left(v_{1} / v_{0}\right)}{L+\lambda(1-2 a)\left(v_{1}-v_{0}\right)} ;$

cf. eqn. (24). Using

$\dot{\gamma}=\frac{3 Q}{2 h_{0}^{3} W} y, N_{1}=2 \eta_{0} \lambda \dot{\gamma}^{2}$ and $Q=2 h_{0} v_{0} W$, 
the critical force can be found (see Appendix B):

$F_{\mathrm{c}}=2 \int_{0}^{h_{11}} N_{1} \mathrm{~d} y=\frac{3 \eta_{0} \lambda Q^{2}}{h_{0}^{3}}$,

from which the vortex size results (combine eqns. (59) and (60)):

$$
\frac{L}{h_{0}}=\frac{2}{3 D e} \ln \beta+D e(2 a-1)(\beta-1) \text {. }
$$

Figure 15 shows the vortex size as a function of the Deborah number for the planar (eqn. (62)) and axisymmetric (eqn. (54)) contraction, for the same value of $\beta$, providing a possible answer to the problem White and Baird [43] referred to.

\subsection{Couette correction for a circular contraction}

The extra pressure drop over a circular contraction with entrance radius $R_{0}$ and length $L_{0}$, exit radius $R_{1}$ and length $L_{1}$, can be expressed as the Couette correction, (see for example Debbaut and Crochet [45]):

$C=\left(\Delta p_{\text {tot }}-\left|\nabla p_{0}\right| L_{0}-\left|\nabla p_{1}\right| L_{1}\right) / 2 \tau_{W}$

where the scaling parameter, the shear stress $\tau_{W}$, is obtained in the fully developed region of the exit $\left(R_{1}\right)$. If a vortex of length $L$ is present, the total pressure drop can be expressed as:

$\Delta p_{\text {tot }}=\left|\nabla p_{0}\right|\left(L_{0}-L\right)+\left|\nabla p_{1}\right| L_{1}+\Delta p_{L}$.

Consequently

$C=\frac{\Delta p_{L}-\left|\nabla p_{0}\right| L}{2 \tau_{W}}$

The vortex size $L$ is given in eqn. (54) for an upper-convected Maxwell model with a constant viscosity, the critical spinning force is found in eqn. (40) and the pressure drop over the vortex can be derived from an overall energy balance, under the assumption that the pressure inside the vortex is isotropic and homogeneous:

$\Delta p_{L}=-\left(\frac{F}{\pi R_{0}^{2}}-\frac{F}{\pi R_{1}^{2}}\right)$.

Combining eqns. (40) and (66), we obtain

$\Delta p_{L}=\frac{16 \lambda \eta v_{0}^{2}}{R_{0}^{2}}\left(\beta^{2}-1\right)$, 


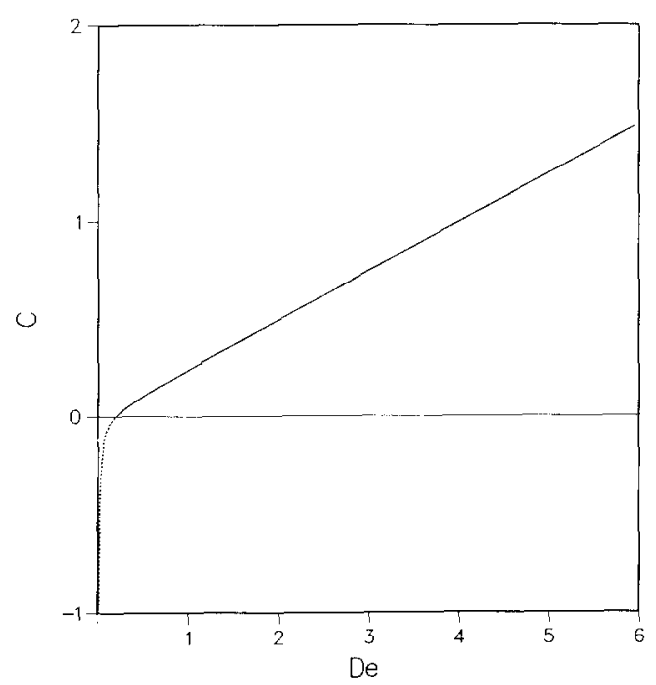

Fig. 18. Couette correction versus Deborah number calculated for an upper-convected Maxwell model in a circular $4: 1$ contraction, eqn. (68).

and from eqns. (54), (65) and (67), we have

$C=\frac{1}{\beta^{3}}\left[D e\left(\beta^{2}-1\right)-\frac{3}{8 D e} \ln \beta\right]$,

with $\beta=\frac{R_{0}}{R_{1}}$

For the $4: 1$ contraction it follows that

$C=\frac{1}{4} D e-\frac{10^{-2}}{D e}$,

which is graphically shown in Fig. 18. Again, unrealistic values are found at extremely low Deborah numbers, owing to the unrealistic constitutive equation used and the fact that the radial velocity gradients are neglected in our analysis.

\section{Discussion}

Via a number of numerical simulations, Debbaut and Crochet recently arrived at the following statement (Ref. 45, p. 177): “We might conclude that a rapidly growing extensional viscosity has a positive effect upon the vortex growth and that $N_{1}$ has the opposite effect". Equation (51) supports this viewpoint. Qualitatively, predictions of the contraction flow problem on the basis of numerical analyses (as in Ref. 45) are correct, but quantitatively 
a gap of at least one order of magnitude, as expressed in the Deborah number or the stress ratio $S_{\mathrm{r}}$, still exists between predictions and experiments, see Ref. 15. This striking difference calls for a simplified analysis as presented here, the main advantage of which may be that insight is obtained into the mechanism behind vortex growth, at least for viscoelastic fluids.

However, there exists an experimental problem here. Apart from other problems with the rheological characterization of the solutions we used, the transient elongational viscosity could not be measured in isothermal experiments, partly because decalin dissolves in silicone oil (which was used to keep the temperature constant and to eliminate gravity forces) and partly because the solutions break during isothermal experiments in tensile tests owing to lack of 'strain hardening' at low extensional rates or low deformations. As a consequence, only data obtained by non-isothermal spinning experiments could be used in order to model the same spinning process, an unsatisfying and moreover unacceptable procedure. Even in the extensive literature on model liquids, we were not able to find a complete set of experimental data for an evaluation of the model. What is needed is the vortex size as a function of Deborah number for well-defined fluids, i.e. fluids with a known shear-dependent first normal stress difference and transient elongational viscosity.

Another interesting experimental difficulty is that both determining properties, elongational viscosity and first normal stress difference, depend strongly on the high end tail of the molecular weight distribution. This showed up in our experiments via the unexpected but pronounced influence of the extruder screw speed on both vortex size and spinning force. From the small drop in intrinsic viscosity measured, pointing to a decrease in molecular weight due to thermal and especially mechanical degradation, these effects cannot be explained if only the averaged molecular weight dependence of the shear viscosity is taken into account. The molecular weight distribution and especially the high end tail of this distribution dominates the extensional flow behaviour. This tail cannot be measured accurately with existing methods such as GPC [1]. An interesting experimental possibility has been proposed by Keller's group [46-48], using the molecular weight dependence of the coil-stretch transition in extensional flows. This method might be combined with our results in order to find the dependence of the first normal stress difference at high shear rates on the molecular weight distribution.

\section{Acknowledgements}

The analytical approximation of viscoelastic spinning was proposed by Herman Wallink. The experimental work was performed by Rob Tesselaar, 
Hans van de Veen, Pascal Pelaers and Helena Derand. All computer simulations and graphics were made by Armand Duysens. The technical support of Gerrit Martens, in the design and construction of the different experimental set-ups, was highly valuable. The research was done at the laboratory of DSM Research. The permission of DSM Research to publish these results is appreciated.

\section{Notation}

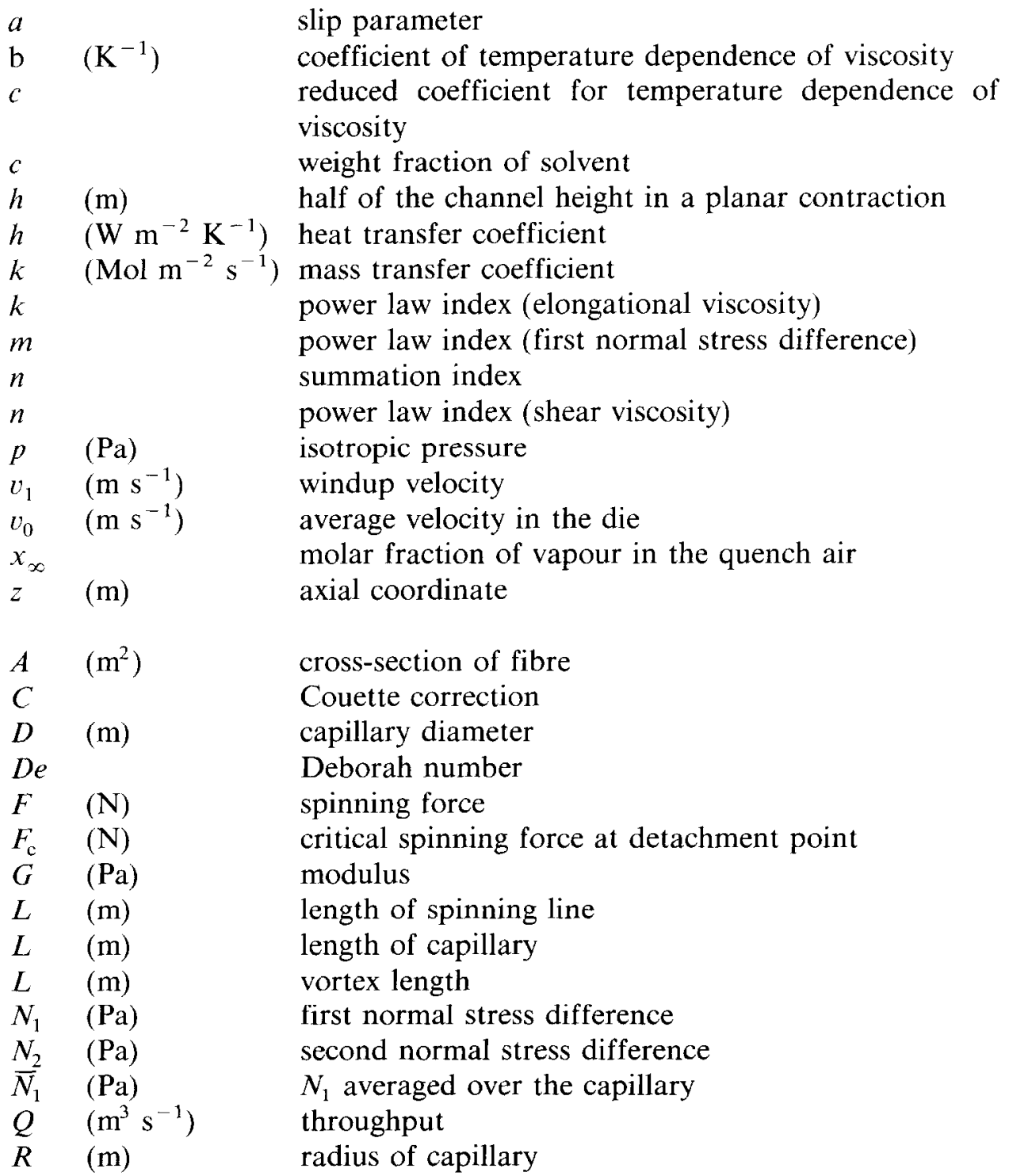




$\begin{array}{ll}T & (\mathrm{~K}) \\ T_{\mathrm{c}} & (\mathrm{K}) \\ T_{0} & (\mathrm{~K}) \\ T_{1} & (\mathrm{~K}) \\ W & (\mathrm{~m})\end{array}$

$\begin{array}{ll}\boldsymbol{L} & \left(\mathrm{s}^{-1}\right) \\ \boldsymbol{D} & \left(\mathrm{s}^{-1}\right) \\ \boldsymbol{\sigma} & (\mathrm{Pa}) \\ \dot{\boldsymbol{\sigma}} & \left(\mathrm{Pa} \mathrm{s} \mathrm{s}^{-1}\right) \\ \dot{\boldsymbol{\sigma}} & \left(\mathrm{Pa} \mathrm{s} \mathrm{s}^{-1}\right)\end{array}$

$\alpha$

$\alpha$

$\beta$

$\gamma \quad\left(\mathrm{N} \mathrm{m}^{-1}\right)$

$\dot{\gamma} \quad\left(\mathrm{s}^{-1}\right)$

$\eta_{\mathrm{e} 0} \quad\left(\mathrm{~Pa} \mathrm{~s}^{k}\right)$

$\eta_{\mathrm{e}} \quad(\mathrm{Pa} \mathrm{s})$

$\eta_{0} \quad\left(\mathrm{~Pa} \mathrm{~s}^{n}\right)$

$\eta \quad(\mathrm{Pa} \mathrm{s})$

$\rho \quad\left(\mathrm{kg} \mathrm{m}^{-3}\right)$

$\psi_{0} \quad\left(\mathrm{~Pa} \mathrm{~s}^{m+1}\right)$

$\sigma_{11} \quad(\mathrm{~Pa})$

$\begin{array}{ll}\sigma_{22} & (\mathrm{~Pa})\end{array}$

$\sigma_{z z} \quad(\mathrm{~Pa})$

$\sigma_{r r} \quad(\mathrm{~Pa})$

$\sigma_{\theta \theta} \quad(\mathrm{Pa})$

$\sigma_{c} \quad(\mathrm{~Pa})$ temperature

crystallization temperature

temperature in the die

temperature on leaving the spinning line

channel width

deformation rate

rate of strain

stress tensor

material derivative

corotational derivative

exponent in prescribed temperature profile

exponent in concentration dependence of elongational viscosity

geometrical contraction ratio

surface tension

shear rate

prefactor for the elongational viscosity

elongational viscosity

prefactor for the shear viscosity

shear viscosity

density

prefactor in power law for $N_{1}(\dot{\gamma})$

extra normal stress in $z$ direction

extra normal stress in $r$ direction

total normal stress in $z$ direction

total normal stress in $r$ direction

total normal stress in $\theta$ direction

critical total stress for pullout to occur

\section{References}

1 G. Eder, H. Janeschitz-Kriegl, S. Liedauer, A. Schausberger, W. Stadlbaur and G. Schlindlauer, J. Rheol., 33 (1989) 805.

2 M.H. Wagner and H.M. Laun, Rheol. Acta, 17 (1978) 138.

3 P.J.R. Leblans, Ph.D. Thesis, University of Antwerp, The Netherlands, 1985.

4 J. Meissner, Chimia, 38 (1984) 35-45; 38 (1984) 65-75.

5 C.J.S. Petrie, Elongational Flows, Pitman, London, 1979.

6 M.A. Matovich and J.R.A. Pearson, Ind. Eng. Chem. Fundam., 8 (1969) 512.

7 M.M. Denn, C.J.S. Petrie and P. Avenas, AIChE J., 21 (1975) 791.

8 I. Mewis and C.I.S. Petrie, Hydrodynamics of spinning polymers, in Encyclopedia of Fluid Mechanics, Gulf Publishing Company, Houston, TX, 1986. 
9 C.J.S. Petrie, Film blowing, blow moulding and thermoforming in: J.R.A. Pearson and S.M. Richardson (Eds.), Computational Analysis of Polymer Processing, Elsevier Applied Science Publishers, London, 1983.

10 E. Fisher, Ph.D. Thesis, IKT Stuttgart, 1981.

11 H. Kaiser, Ph.D. Thesis, IKT Stuttgart, 1990.

12 J. Mewis and G. de Cleyn, AIChE J., 28 (1982) 900.

13 J. Sampers and P.J.R. I eblans, I. Non-Newtonian Fluid Mech., 30 (1988) 325.

14 R. Keunings, M.J. Crochet and M.M. Denn, Ind. Eng. Chem. Fundam., 22 (1983) 347.

15 B. Debbaut, J.M. Marchal and M.J. Crochet, J. Non-Newtonian Fluid Mech., 29 (1988) 119.

16 F.N. Cogswell, Polym. Eng. Sci., 12 (1972) 64.

17 D.M. Binding, J. Non-Newtonian Fluid Mech., 27 (1988) 173.

18 R. Keunings and M.J. Crochet, J. Non-Newtonian Fluid Mech., 14 (1984) 279.

19 J. Non-Newtonian Fluid Mech., 20 (1986), special issue.

20 D.V. Boger and A.V. Rama Murthy, Rheol. Acta, 11 (1972) 61.

21 P.J. Cable and D.V. Boger, AIChE J., 24 (1978) 869.

22 R.E. Evans and K. Walters, J. Non-Newtonian Fluid Mech., 20 (1986) 11.

23 D.V. Boger, D.U. Hur and R.J. Binnington, J. Non-Newtonian Fluid Mech., 20 (1986) 31.

24 D.V. Boger, Annu. Rev. Fluid Mech., 19 (1987) 157, references p. 170.

25 K. Walters, in: A.S. Lodge, M. Renardy and J.A. Nohel (Eds.), Viscoelasticity and Rheology, Academic Press, London, 1985, pp. 47-79.

26 P. Smith and P.J. Lemstra, DSM/Stamicarbon, US Patents 4,344,908; 4,422,933; $4,430,383 ; 4,436,689$.

27 P. Smith, P.J. Lemstra and H.C.J. Booij, J. Polym. Sci., Polym. Phys. Ed., 19 (1981) 877.

28 P.J. Lemstra, C.W.M. Bastiaansen and H.E.H. Meijer, Angew. Makromol. Chem., 145/146 (1986) 343.

29 T. Sridhar and R.K. Gupta, J. Non-Newtonian Fluid Mech., 30 (1988) 285.

30 D.M. Binding and K. Walters, J. Non-Newtonian Fluid Mech., 30 (1988) 233.

31 C.J.S Petrie, Elongational Flows, Pitman, London, 1979, p. 28 and references cited therein.

32 C.J.S. Petrie, Elongational Flows, Pitman, London, Pitman, 1979, p. 65.

33 II.A. Wallink, Private communications.

34 C.J.S. Petrie, Elongational Flows, Pitman, London, 1979, p. 63.

35 C.J.S. Petrie, in: C. Klason and J. Kubat (Eds.), Proc. VIIth Int. Congr. on Rheology, Gothenburg, 1976, pp. 446-447.

36 G.R. Zeichner, Spinnability of viscoelastic fluids, M.Ch.E. Thesis, University of Delaware, Newark, DE, 1973.

37 C.J.S. Petrie, Elongational Flows, Pitman, London, 1979, pp. 56-70, pp. 162-168.

38 Y. Ohzawa and Y. Nagano, J. Appl. Polym. Sci., 13 (1969) 257.

39 Y. Ohzawa and Y. Nagano, J. Appl. Polym. Sci., 14 (1970) 1879.

40 M.J. Crochet, Polyflow, A Finite Flement Program for the Simulation of Viscoelastic Flow, Chapman and Hall, Louvain-la-Neuve, 1982.

41 D.V. Boger and M.M. Denn, J. Non-Newtonian Fluid Mech., 6 (1980) 163.

42 D.V. Boger and R.R. Huilgol, Trans. Soc. Rheol., 21 (1977) 447.

43 S.A. White and D.G. Baird, J. Non-Newtonian Fluid Mech., 29 (1988) 245.

44 W.P. Raiford, L.M. Quinzani, P.J. Coates, R.C. Amstrong and R.A. Brown, J. Non-Newtonian Fluid Mech., 32 (1989) 39.

45 B. Debbaut and M.J. Crochet, J. Non-Newtonian Fluid Mech., 30 (1988) 169.

46 A. Keller and J.A. Odell, Colloid Polym. Sci., 263 (1985) 181. 
47 A. Keller, A.J. Müller and J.A. Odell, Progr. Colloid Polym. Sci., 75 (1987) 179.

48 A.J. Müller, J.A. Odell and A. Keller, Macromolecules, in press.

49 K. Walters, Rheometry, Chapman and Hall, London, 1975.

50 A.S. Lodge, J. Rheol., 33 (1989) 821.

\section{Appendix A}

Is $[(v \cdot \nabla) \sigma \stackrel{?}{=} \sigma \cdot(\nabla v)]$ true, for steady uniaxial extensional flows?

In spinning, the stress and rate of deformation tensors read

$\boldsymbol{\sigma}=\left[\begin{array}{ccc}\sigma_{11} & 0 & 0 \\ 0 & \boldsymbol{\sigma}_{22} & 0 \\ 0 & 0 & \sigma_{33}\end{array}\right]$ and $\nabla \boldsymbol{v}=\left[\begin{array}{ccc}\frac{\partial v_{z}}{\partial z} & 0 & 0 \\ 0 & -\frac{1}{2} \frac{\partial v_{z}}{\partial z} & \\ 0 & 0 & -\frac{1}{2} \frac{\partial v_{z}}{\partial z}\end{array}\right]$

Using eqn. (A.1) the equality reads

$v_{z} \frac{\partial \sigma_{11}}{\partial z} \stackrel{?}{=} \sigma_{11} \frac{\partial v_{z}}{\partial z}$

$v_{z} \frac{\partial \sigma_{22}}{\partial z} \stackrel{?}{=} \sigma_{22}-\frac{1}{2} \frac{\partial v_{z}}{\partial z}$.

Summation of eqns. (A.2) and (A.3) gives, after some rearrangements,

$v_{z} \partial\left(\sigma_{11}-\sigma_{22}\right) / \partial z=\left(\sigma_{11}-\sigma_{22}\right) \frac{\partial v_{z}}{\partial z}+\frac{3}{2} \sigma_{22} \frac{\partial v_{z}}{\partial z}$.

In spinning we have

$\sigma_{11}-\sigma_{22}=\frac{F}{\pi R^{2}}$

and

$v_{z}=\frac{Q}{\pi R^{2}}$,

where $F$ and $Q$ are constants.

Combination of eqns. (A.4), (A.5) and (A.6) shows that the equality is fulfilled when the last term on the right-hand side of eqn. (A.4) is zero, that is when $\sigma_{22}$ is neglected with respect to $\sigma_{11}$. 


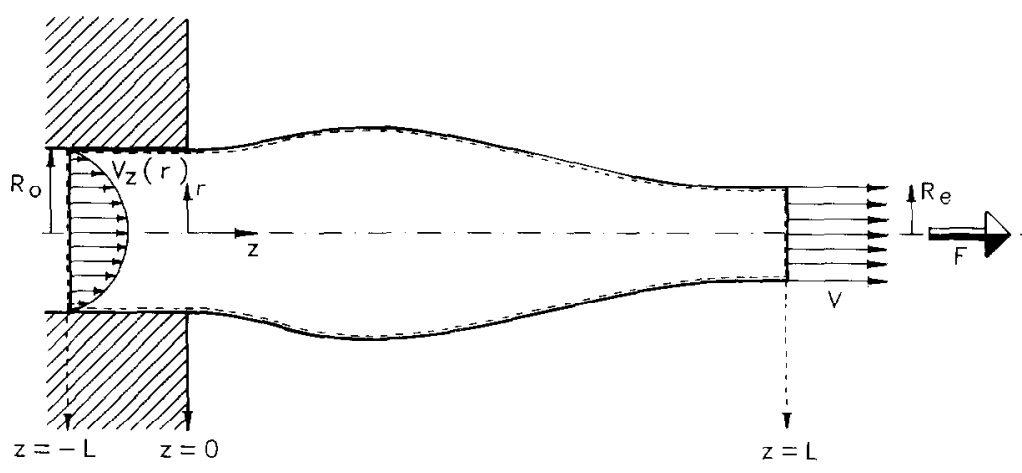

Fig. 19. Integral momentum balance over the dashed contour line.

\section{Appendix B}

Stress balance at the detachment point

\section{Circular flow}

The spinning force at which pullout starts can be calculated from an integral momentum balance, in a way analogous to that described by Boger and Huilgol [42] and Boger and Denn [41]. The momentum balance is made up over the dotted contour in Fig. 19.

The momentum balance results in

$$
\begin{aligned}
\rho \pi R_{\mathrm{e}}^{2} v^{2}-\left.\int_{0}^{R_{0}} v_{z}^{2}(r)\right|_{z=L} \rho 2 \pi r \mathrm{~d} r= & -\left[\int_{0}^{R_{0}} \sigma_{z z} 2 \pi r \mathrm{~d} r\right]_{z=-L}+F \\
& +\int_{z=-L}^{0} \sigma_{r z}\left(R_{0}\right) 2 \pi R_{0} \mathrm{~d} z .
\end{aligned}
$$

When viscometric or nearly viscometric flow is assumed at the exit of the capillary, the limit of eqn. (B.1) for $L \rightarrow 0$ can be taken. When inertia is also neglected, eqn. (B.1) reduces to

$$
\left.\int_{0}^{R_{0}} \sigma_{z z}\right|_{z=0} 2 \pi r \mathrm{~d} r=F
$$

The normal stress $\sigma_{\mathrm{zz}}$ at the exit can be expressed in the first and second normal stress differences.

One of the three components of the equation of motion in cylindrical coordinates for a cylindrical symmetric flow is given by

$$
\frac{\partial \sigma_{r r}}{\partial r}+\frac{1}{r}\left(\sigma_{r r}-\sigma_{\theta \theta}\right)=0 .
$$


This equation can be solved for $\sigma_{\mathrm{rr}}$ :

$\sigma_{r r}(r, z)=\sigma_{r r}(0, z)-\int_{0}^{r} \frac{N_{2}}{r} \mathrm{~d} r$

or

$\sigma_{r r}(r, z)=\sigma_{r r}\left(R_{0}, z\right)-\int_{R_{0}}^{r} \frac{N_{2}}{r} \mathrm{~d} r$,

where the second normal stress difference $N_{2}$ is introduced,

$N_{2}(r, z)=\sigma_{r r}(r, z)-\sigma_{\theta \theta}(r, z)$.

Using the following definition of the first normal stress difference,

$N_{1}(r, z)=\sigma_{z z}(r, z)-\sigma_{r r}(r, z)$,

eqn. (B.5) can be rewritten

$\sigma_{z z}(r, z)=N_{1}(r, z)-\sigma_{r r}\left(R_{0}, z\right)-\int_{R_{0}}^{r} \frac{N_{2}}{r} \mathrm{~d} r$.

This can be substituted into eqn. (B.2) for the spinning force:

$\int_{0}^{R_{0}} N_{1} 2 \pi r \mathrm{~d} r-\int_{0}^{R_{0}} \sigma_{r r}\left(R_{0}\right) 2 \pi r \mathrm{~d} r-\int_{0}^{R_{0}}\left(\int_{x=R_{0}}^{r} \frac{N_{2}}{x} \mathrm{~d} x\right) \mathrm{d} r=F$.

When the last integral on the left-hand side is evaluated by integrating by parts, a relation between the normal stress at the wall $\sigma_{\mathrm{rr}}\left(R_{0}, 0\right)$ at the exit of the capillary and the spinning force is found:

$\sigma_{r r}\left(R_{0}, 0\right)=\frac{2}{R_{0}^{2}} \int_{0}^{R_{0}}\left(N_{1}+\frac{N_{2}}{2}\right) r \mathrm{~d} r-\frac{F}{\pi R_{0}^{2}}$.

The condition for pullout to occur is that the stress normal to the wall at the exit of the capillary should be zero, so

$\sigma_{r r}\left(R_{0}, 0\right)=0$.

Combining eqns. (B.10) and (B.11) yields the critical spinning force at which pullout will start.

$F_{\mathrm{c}}=2 \pi \int_{0}^{R_{0}}\left(N_{1}+\frac{N_{2}}{2}\right) r \mathrm{~d} r$

\section{Planar flow}

For a planar flow in the $z$ direction of infinite width and height $2 h(-h \leq$ $y \leq h$ ), eqn. (B.2) reads

$$
F=\left.2 \int_{0}^{h} \sigma_{z z} \mathrm{~d} z\right|_{z=0}
$$


with $F$ in pascal metres. The equations of motion reduce to

$\frac{\partial \sigma_{y y}}{\partial y}=0 \quad$ consequently $\quad \sigma_{y y}=c(z)$,

$\frac{\partial \sigma_{z z}}{\partial z}+\frac{\partial \sigma_{y z}}{\partial y}=0$

(cf. eqn. (B.3)). With the definition of the first normal stress difference,

$N_{1}(y, z)=\sigma_{z z}(y, z)-\sigma_{y y}(y, z)$,

eqn. (B.13) can be rewritten

$F=2 \int_{0}^{h}\left[N_{1}(y, 0)+\sigma_{y y}(y, 0)\right] \mathrm{d} y$.

Because of eqn. (B.14) we have $\sigma_{\mathrm{yy}}(y, 0)=\sigma_{\mathrm{yy}}(h, 0)$, and consequently

$F=2 h \sigma_{y y}(h, 0)+2 \int_{0}^{h} N_{1}(y, 0) \mathrm{d} y$,

which leads to the critical force, $F_{\mathrm{c}}$, if combined with the condition for pullout $\sigma_{y y}(h, 0)=0$ :

$F_{c}=2 \int_{0}^{h} N_{1}(y, 0) \mathrm{d} y$.

(cf. eqn. (B.12)).

\section{Appendix C}

Measurement of the first normal stress difference from pullout measurements

By measuring the force at which pullout starts versus capillary diameter or versus flow rate for a long capillary it seems possible to determine the shear rate dependence of the first normal stress difference. To be able to do this, the second normal stress difference should be negligible relative to the first. Under this condition the expression for the critical force reads

$F_{\mathrm{c}}=\int_{0}^{R} 2 \pi r N_{1} \mathrm{~d} r$.

By differentiating this expression with respect to the radius $R$ it is found that

$N_{1}\left(\dot{\gamma}_{R}\right)=\frac{1}{2 \pi R} \frac{\mathrm{d} F_{\mathrm{c}}}{\mathrm{d} R}$,

where $\dot{\gamma}_{R}$ is the shear rate at the wall. 
Using this equation to determine the shear rate dependence of $N_{1}$ would involve a rather laborious measuring procedure because the shear rate as well as the capillary diameter should be varied. A more convenient expression can be obtained with a Rahinowitsch-like procedure [49]. For tube flow, the shear stress at the wall can be related to the shear stress at radius $r$,

$\frac{\tau(r)}{r}=\frac{\tau_{R}}{R}$.

Substitution of eqn. (C.3) into eqn. (C.1) yields

$F_{\mathrm{c}}=\int_{\tau=0}^{\tau_{w}} \frac{2 \pi R^{2}}{\tau_{W}^{2}} \tau N_{1} \mathrm{~d} \tau$

or

$\frac{F_{\mathrm{c}} \tau_{W}^{2}}{2 \pi R^{2}}=\int_{\tau=0}^{\tau_{W}} \tau N_{1} \mathrm{~d} \tau$

When eqn. (C.5) is differentiated with respect to $\tau_{W}$ the desired expression is obtained.

$\frac{\mathrm{d}}{\mathrm{d} \tau_{W}}\left(\frac{F_{\mathrm{c}} \tau_{W}^{2}}{2 \pi R^{2}}\right)=\tau_{W} N_{1}\left(\dot{\gamma}\left(\tau_{W}\right)\right)$

or

$N_{1}\left(\dot{\gamma}_{W}\right)=\frac{1}{\tau_{W}} \frac{\mathrm{d}}{\mathrm{d} \tau_{W}}\left(\frac{F_{\mathrm{c}} \tau_{W}^{2}}{2 \pi R^{2}}\right)$.

When the critical spinning force is measured versus the shear stress at the wall (i.e. flow rate) the first normal stress difference versus shear rate results after a differentiation operation. Expression (C.7) can be written in a form which is independent of the capillary diameter by introducing the critical spinning tension $\sigma_{\mathrm{c}}$ as

$\sigma_{\mathrm{c}}=F_{\mathrm{c}} / \pi R^{2}$,

resulting in

$N_{1}\left(\dot{\gamma}_{W}\right)=\frac{1}{2 \tau_{W}} \frac{\mathrm{d}}{\mathrm{d} \tau_{W}}\left(\sigma_{\mathrm{c}} \tau_{W}^{2}\right)$

By this procedure the first normal stress differences at high shear rates might be measured, thus providing a possible alternative for Lodge's method [50]. 